\title{
Epidemiology incidence and mortality worldwide common cancers in males and their relationship with the human development index (HDI): An ecological study updated in the world
}

\author{
Elham Goodarzi, ${ }^{a}$ Reza Beiranvand, ${ }^{\text {b }}$ Alireza Mosavi-Jarrahi, ${ }^{, d}$ Hasan Naemi, ${ }^{\text {Z Zaher Khazaeif }}$
}

aSocial Determinants of Health Research Center, Lorestan University of Medical Sciences, Khorramabad, Iran.
bSchool of Medicine, Dezful University of Medical Sciences, Dezful, Iran.
‘Editor-in-Chief, Asia Pacific Journal of Cancer Prevention, Tehran, Iran.
'Department of Epidemiology, Faculty of Health Sciences, Simon Fraser University, BC, Canada.
eIranian Research Center on Healthy Aging, Sabzevar University of Medical Sciences, Sabzevar, Iran.
fDepartment of Epidemiology, School of Public Health, Ilam University of Medical Sciences, Ilam, Iran.
Correspondence to: Zaher Khazaei (Zaherkhazaei@yahoo.com)
(Submitted: 12 October 2019 - Revised version received: 28 October 2019 - Accepted: 12 November 2019 - Published online: 26 December 2019)

This article provides a status report on the global burden of common cancers in male worldwide using the GLOBOCAN 2018 estimates of cancer incidence and mortality.

Based on the results of cancer records in 2018,9,456,418 cases of malignancy were recorded in males, with lung cancer (1,368,524 cases, $14.5 \%)$, prostate cancer $(1,276,106$ cases, $13.5 \%)$, colorectal cancer $(1,026,215$ cases, $10.9 \% \%)$, stomach cancer $(683,754$ cases, $7.2 \%)$, and liver cancer $(596,574$ cases, $6.3 \%)$ were the five most common cancers in men worldwide. The total number of deaths due to cancer in humans in 2018 is 5,385,640. The five causes of death due to cancer in men worldwide are lung cancer $(1,184,947$ cases, $22 \%)$, liver (548,375 cases, $2.10 \%)$, stomach cancer (513,555 cases, 9.5\%), colorectal cancer (484,224 cases, 9\%), and prostate cancer (358,989 cases, 7.7\%). Our results showed that there was a positive correlation between the incidence of lung cancer $(R=0.629, P<0.0001)$, prostate cancer ( $R=$ $0.534, P<0.0001)$, colorectal cancer $(R=0.745, P<0.0001)$, and stomach cancer $(R=0.268, P<0.001)$ with human development index (HDI) index, while there was no significant relationship between liver cancer and the HDI $(R=0.079, P>0.05)$. The results also showed that there was a positive and significant correlation between mortality from lung cancer $(R=0.632, P<0.0001)$ and colorectal cancer $(R=0.627$, $P<0.0001)$ with $\mathrm{HDI}$, whereas this correlation was negative for prostate cancer $(R=-0.187, P<0.01)$.

Keywords: Common cancer, incidence, mortality, males, HDI

\section{Introduction}

During the last century, there have been serious changes in the incidence of diseases. Reducing the burden of contagious diseases and increasing the incidence, prevalence, and mortality of non-communicable and chronic diseases and incidents are the most significant of these changes. ${ }^{1-3}$ This is not the case for developed countries, and developing countries have been dramatically affected by these $e^{4,5}$ changes. Reports indicate that in the years up to 2015 , about $45 \%$ of all deaths in the world have been caused by non-communicable diseases. In the meantime, cancer has a $10 \%$ share. $^{6}$

Cancer is a multifactorial disease. In various combinations, these can cause cancer. Some of these factors affect the genetic structure of the body, while others cause cancer in people who have genetic infrastructure prone to mutation. ${ }^{7,8}$ Some of the risk factors for cancer include: tobacco consumption, infectious agents, alcohol consumption, reproductive factors such as hormones, nutrition, obesity, insufficient mobility, ionizing radiation, sunlight and ultraviolet radiation, electromagnetic waves, occupational exposures, environmental pollution such as fungi and genetic susceptibility. ${ }^{7,9-11}$ A descriptive epidemiological survey of cancer can help to better understand the cancer etiology to develop preventive strategies, as well as to plan for health systems, diagnosis, and treatment of the $\mathrm{e}^{7,12}$ disease. It will also be useful to acknowledge the role of cancer in morbidity and mortality. Determining the pattern of different cancer types is the first step in planning and coordinating national cancer control. The purpose of this study is to investigate the epidemiology of common cancers in men and their relationship with the development index in 2018 based on GLOBOCAN 2018 data.

\section{Data Sources and Methods:}

Caution must be exercised when interpreting these estimates, given the limited quality and coverage of cancer data worldwide at present, particularly in low- and middle-income countries. IARC's approach is not only to evaluate, compile, and use the data from the Agency's collaborators in these estimates but also to work alongside national staffs to improve local data quality, registry coverage, and analytical capacity. The clear need for investment in population-based cancer registration in low- and middle-income countries led to the launch of the Global Initiative for Cancer Registry Development (GICR), coordinated by IARC. The goal of the GICR is to inform cancer control through defined improvements in the coverage, quality, and use of population-based cancer registration data worldwide. A summary of the steps used to generate the current set of cancer incidence, mortality, and prevalence estimates is provided below. The methods of estimation are country-specific, and the quality of the national estimates depends on the coverage, accuracy, and timeliness of the recorded incidence and mortality data in a given country.

\section{Incidence}

The methods used to estimate the sex- and age-specific incidence rates of cancer in a specific country fall into the following 
broad categories, in order of priority: (1) Observed national incidence rates were projected to 2018 (45 countries). (2) The most recently observed incidence rates (national or regional) were applied to the 2018 population (50 countries). (3) Rates were estimated from national mortality data by modeling, using mortality-to-incidence ratios derived from cancer registries in that country (14 countries). (4) Rates were estimated from national mortality estimates by modeling, using mortality-to-incidence ratios derived from cancer registries in neighboring countries (37 countries). (5) Age- and sex-specific national incidence rates for all cancers combined were obtained by averaging overall rates from neighboring countries. These rates were then partitioned to obtain the national incidence for specific sites using available cancer-specific relative frequency data (7 countries). (6) Rates were estimated as an average of those from selected neighboring countries (32 countries).

\section{Mortality}

The methods used to estimate the sex- and age-specific mortality rates of cancer in a specific country fall into the following broad categories, in order of priority: (1) Observed national mortality rates were projected to 2018 (81 countries). (2) The most recently observed national mortality rates were applied to the 2018 population (20 countries). (3) Rates were estimated from the corresponding national incidence estimates by modeling, using incidence-to-mortality ratios derived from cancer registries in neighboring countries (81 countries). (4) Rates were estimated as an average of those from selected neighboring countries ( 3 countries). ${ }^{13-15}$

\section{Human Development Index}

Human development index (HDI) is a compound index of indices in three dimensions: life expectancy, degree of studies, and dominance over required sources for a proper sensible life. All the groups and regions which have had a remarkable progress in all HDI components have developed more rapidly in comparison with low or moderate HDI countries. As this index says, the world is unequal because national average

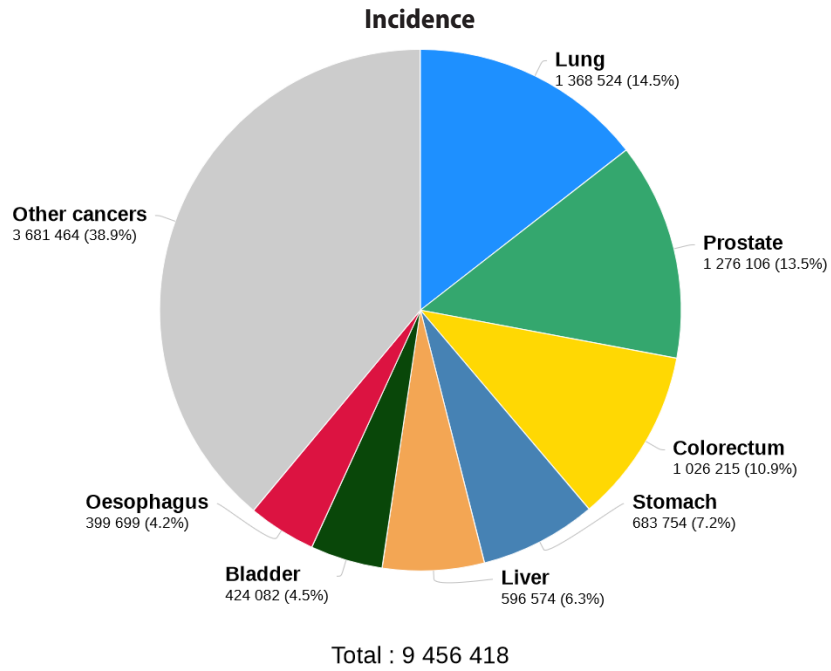

hides most of the different experiences in human's life. There exist a lot of inequalities in northern and southern countries. Income inequality has risen inside every country and also between many countries. ${ }^{16-19}$

\section{Results}

\section{Distribution of Cases and Deaths by World Region and Cancer Type}

Based on the results of cancer records in 2018, 18,078,957 cases of cancer were recorded in both sexes, of which $9,456,418$ cases were in males. The results showed that lung cancer $(1,368,524$ cases, $14.5 \%)$, prostate cancer $(1,276,106$ cases, $5.13 \%)$, colorectal cancer (CRC) $(1,026,215$ cases $9.10 \%)$, stomach cancer $(683,754$ cases $2.7 \%)$, and liver cancer $(596,574$ cases $6.6 \%)$ are the five most common cancers in men worldwide. The total number of deaths due to cancer in 2018 was 9,555,027, of which 5,385,640 cases were men. The results showed that five causes of death due to cancer in men worldwide were related to pulmonary cancers $(1,184,947,22 \%)$, liver (548,375 cases, $2.10 \%)$, stomach $(513,555$ cases, $9.5 \%)$, colorectal $(484,224$ cases, $9 \%)$, and prostate (358,989 cases, 7.7\%) (Fig. 1). Figure 2 shows the most common cancer and the most common cause of death due to cancer in each country (Fig. 2).

\section{Cancer Incidence and Death Rates by World Region}

The results showed that approximately half of the cancers in men $(4,656,551$ cases, $49.2 \%)$ were in Asia, followed by Europe (2,247,518 cases, $23.8 \%)$, and North America (1,274,306 cases, $13.5 \%)$. Of the total deaths due to cancer in men, $3,231,463$ $(60 \%)$ are in Asia, 1,085,592 (20.2\%) in Europe, and 367,738 (6.8\%) in North America (Fig. 3).

\section{Cancer Incidence and Death Rates by World Region}

The results showed that the highest incidence rates in the different regions of the world was related to Australia and New Zealand (527.500 in 100,000), North America $(387 / 100,000)$,

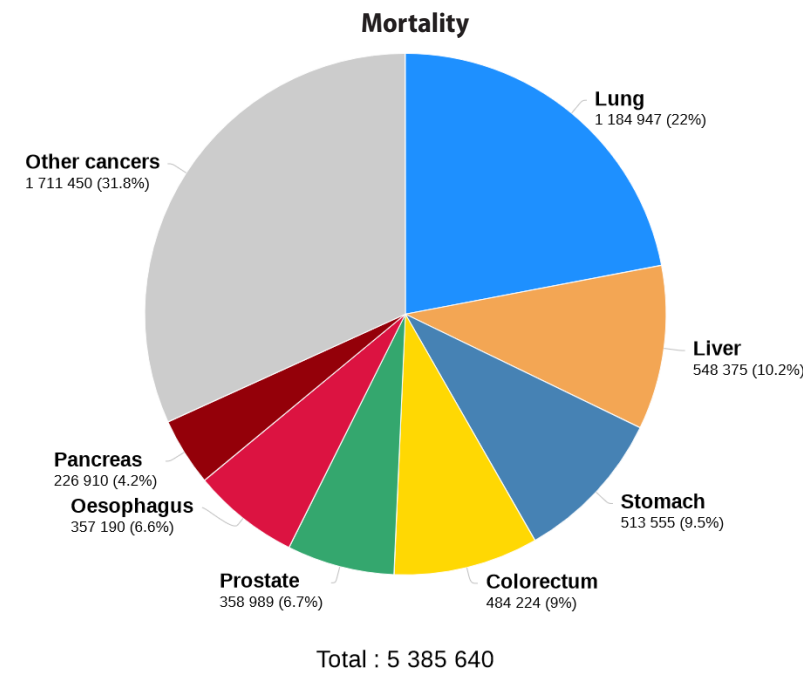

Fig. 1 Pie charts present the distribution of cases and deaths for the seven most common cancers for males in 2018 in worldwide among all ages.

[Source: GLOBOCAN 2018] 
A) Incidence Rate

Top cancer per country, estimated age-standardized incidence rates (World) in 2018, males, all ages
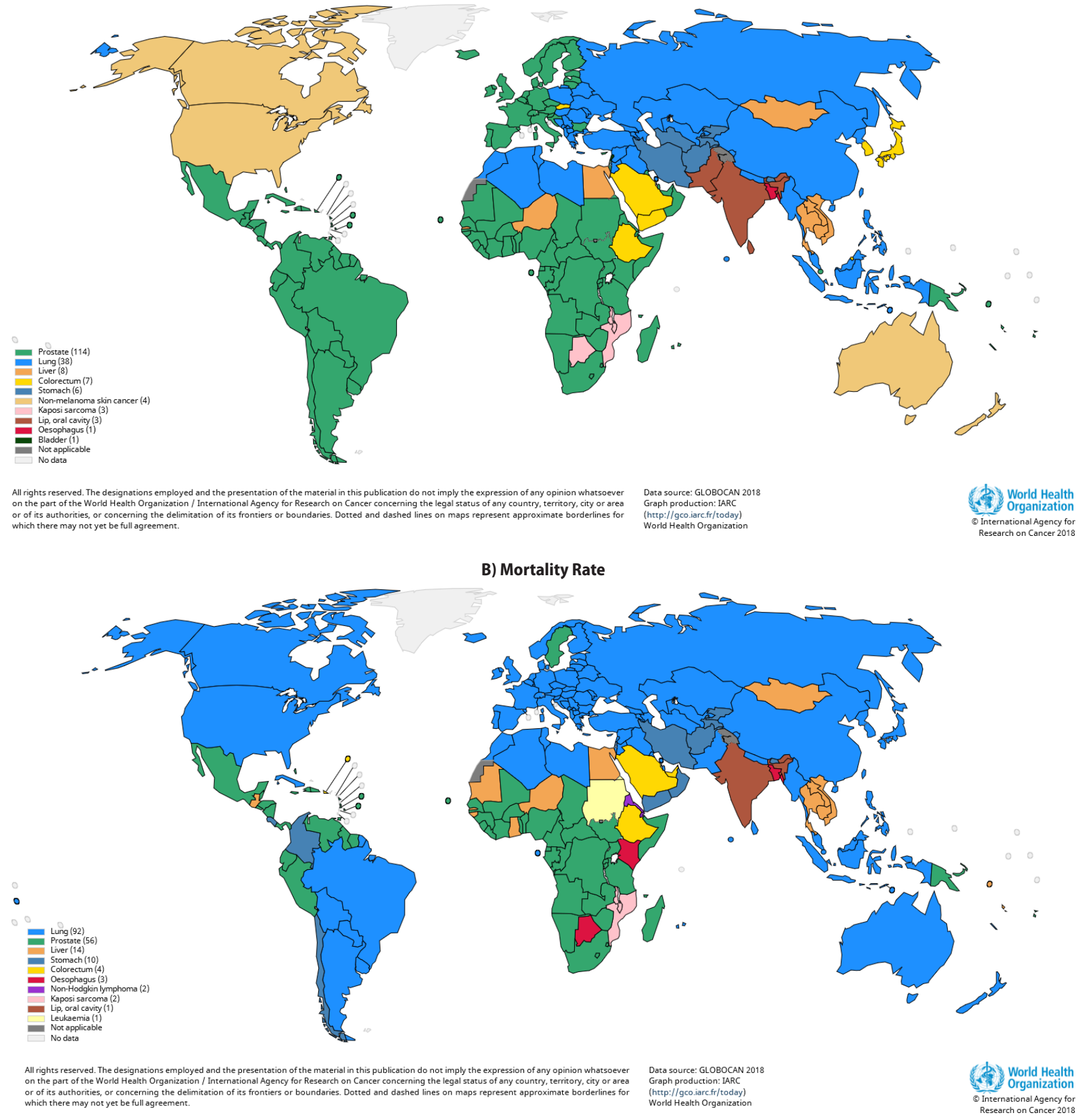

Fig. 2 Global map presenting top cancer country, age-standardized (A) Incidence and (B) Mortality rates by world countries in males for all cancers among all ages in 2018.

Source: GLOBOCAN 2018.

and Western Europe (363/100,000), with the highest mortality rates related to Central and Eastern Europe (171 per 100,000), Eastern Asia (159.6 per 100,000), and Southern Africa (14.4 per 100,000) (Fig. 4).

\section{Cancer Incidence and Mortality Patterns by the 4-tier HDI}

In low/medium HDI areas, the highest incidence of malignancy in men was related to lung cancer $(11.8 / 100,000)$, prostate cancer $(11.4 / 100,000)$, and lip, oral cavity $(8.7 / 100,000)$, while lung cancer (40.4 per 100,000), prostate (37.4 per 100,000), and colorectal $(30.5$ per 100,000$)$ had the highest incidence in high/ very high HDI areas (Fig. 5).

\section{Lung Cancer}

Lung cancer is still the deadliest and most costly cancer in the world, and its mortality rate is three times more than deaths from prostate cancer and nearly twice as high as deaths from 


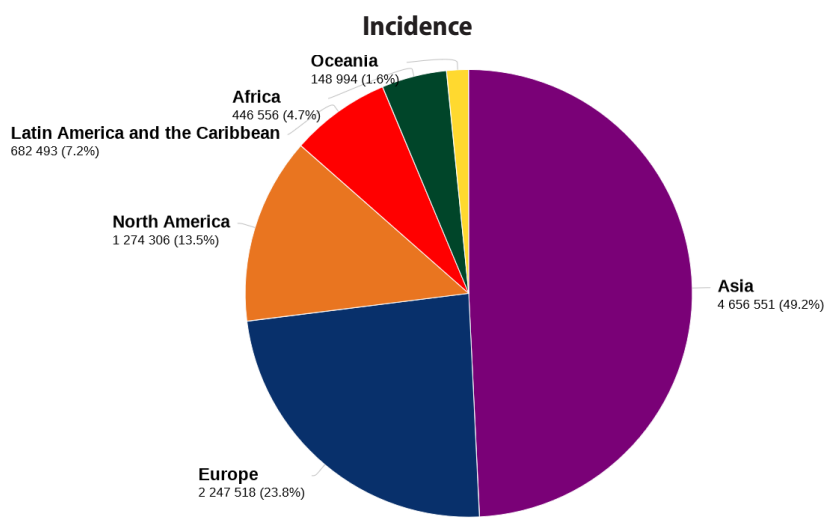

Total : 9456418

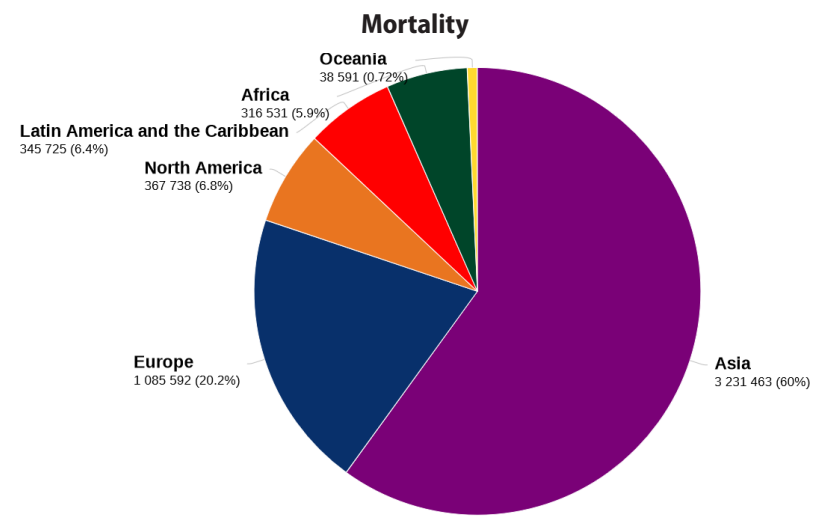

Total : 5385640

Fig. 3 Pie charts present the distribution of cases and deaths by continent in $\mathbf{2 0 1 8}$ for males among all ages.

Source: GLOBOCAN 2018.

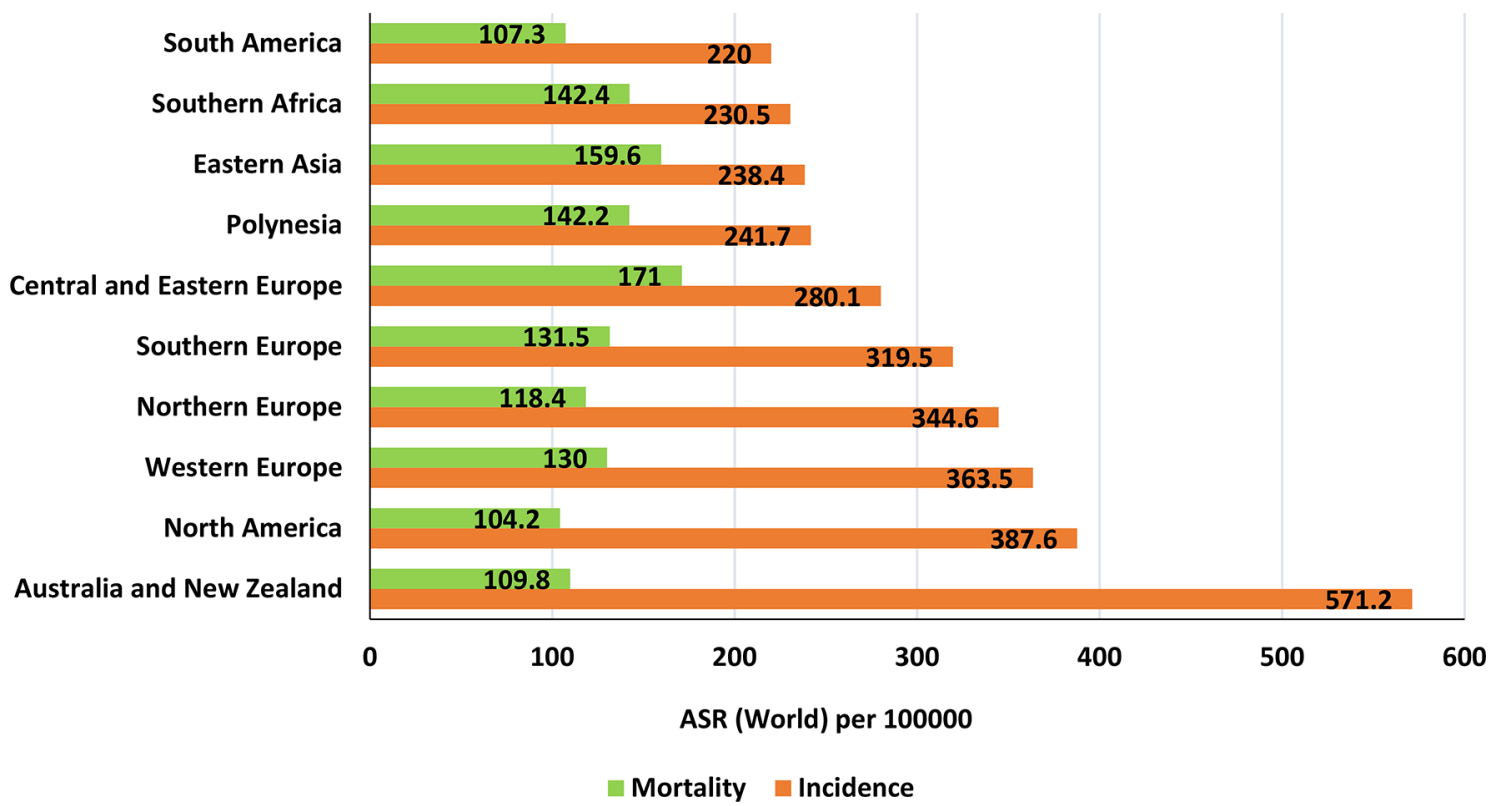

Fig. 4 Bar chart of region-specific incidence and mortality age-standardized rates by world area for males (all cancers) in 2018. Source: GLOBOCAN 2018.

breast cancer in women. Lung cancer currently accounts for $32 \%$ of men's cancer deaths and $20 \%$ of deaths from cancer in women. Today, the incidence of this cancer has a significant reduction in men (from 87 to 63 per 100,000 people), while its rate in women (from 4.1 to 9.9 per 100,000 people) has shown a dramatic increase. According to various studies, the incidence of lung cancer in developed countries is 1.5-2.3 times higher than that of the less developed countries in any age group. Lung cancer is affected by many factors such as environmental and behavioral factors, one of the most important of which is smoking cigarettes. The risk of lung cancer in smokers is 20 times higher than non-smokers. More than $80 \%$ of the lung cancer in the western population is attributed to smoking, which can be prevented through tobacco control.

According to cancer results recorded in 2018, lung cancer has the highest incidence and mortality among the world's cancers in both sexes with 2,093,876 new cases (with 12.22 per
$100,000)$ and 176,1007 deaths (19.88 per 100,000). The highest incidence of lung cancer according to world areas in men was Micronesia (54.1 per 100,000), Polynesia (52 per 100,000), and Central and Eastern Europe (49.3 per 100,000), with the highest mortality rate for Micronesia (7.51 at 100,000), Central and Eastern Europe (44/100000), and Polynesia (2.43 at 100,000) (Fig. 6). According to cancer records in 2018, Hungary (77.4 per 100,000$)$, Serbia (71.6 per 100,000), and Turkey $(70.6$ per 100,000$)$ have the highest incidence of lung cancer in men (Table 1, Fig. 7). Studies show that the number of deaths from lung cancer in most developed countries is mainly due to the aging population and, in the less developed countries, the gradual increase in the use of cigarettes and tobacco by control of which, mortality rates could be reduced.

According to the results of 2018, the highest mortality rates for lung cancer were in Turkey $(68.6 \%$ in 100,000$)$, Hungary $(62.9 \%$ in 100,000$)$, and Serbia $(61.1$ in 100,000$)$ (Table 2). 


\section{High/Very high HDI}

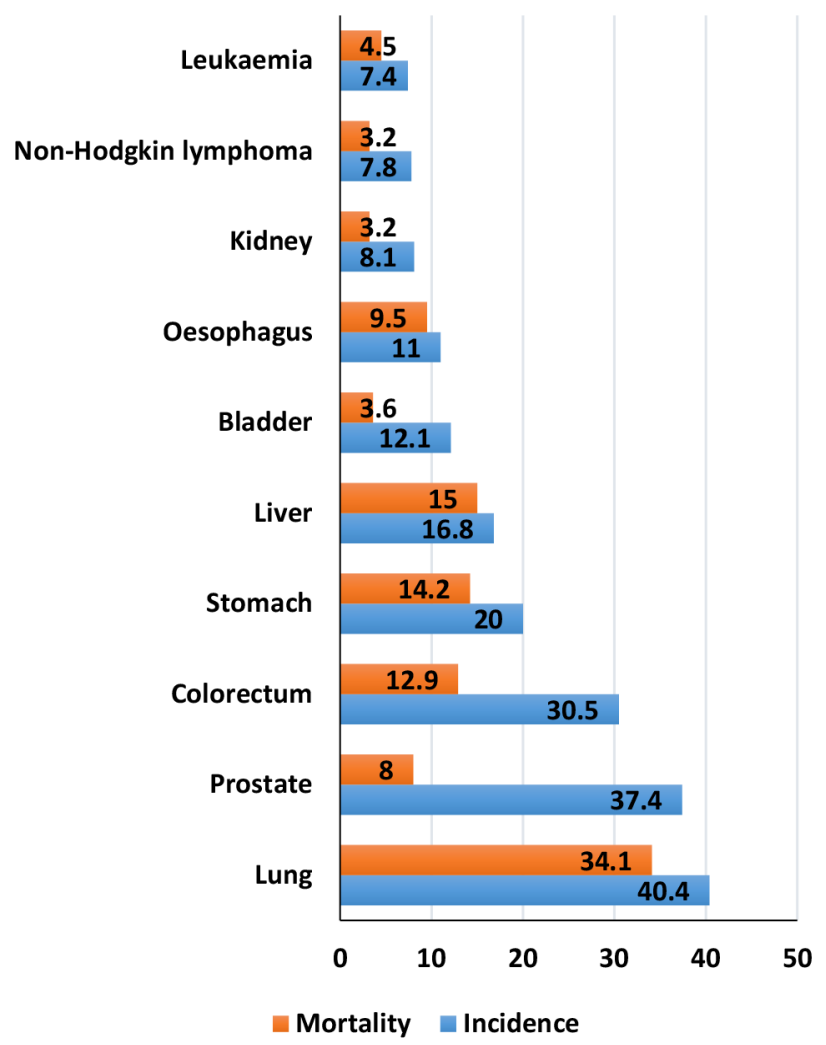

\section{Low/Medium HDI}

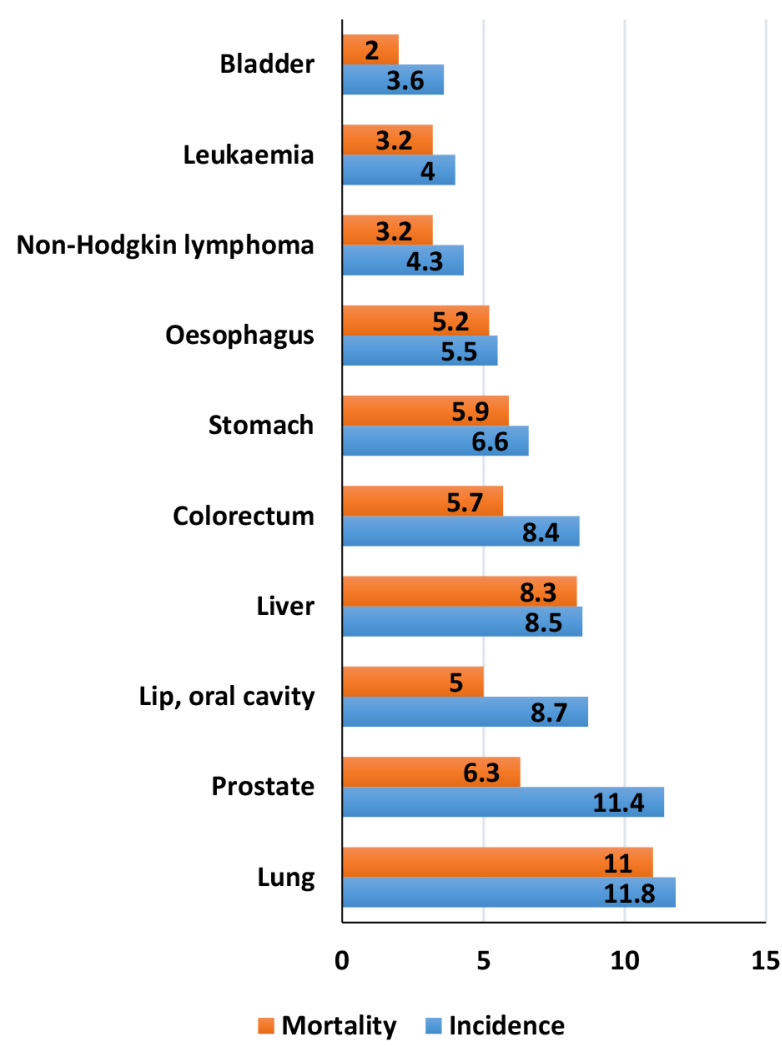

Fig. 5 Bar charts of incidence and mortality age-standardized rates in high/very-high human development index regions versus low/ medium human development index regions.

Source: GLOBOCAN 2018

\section{Lung}

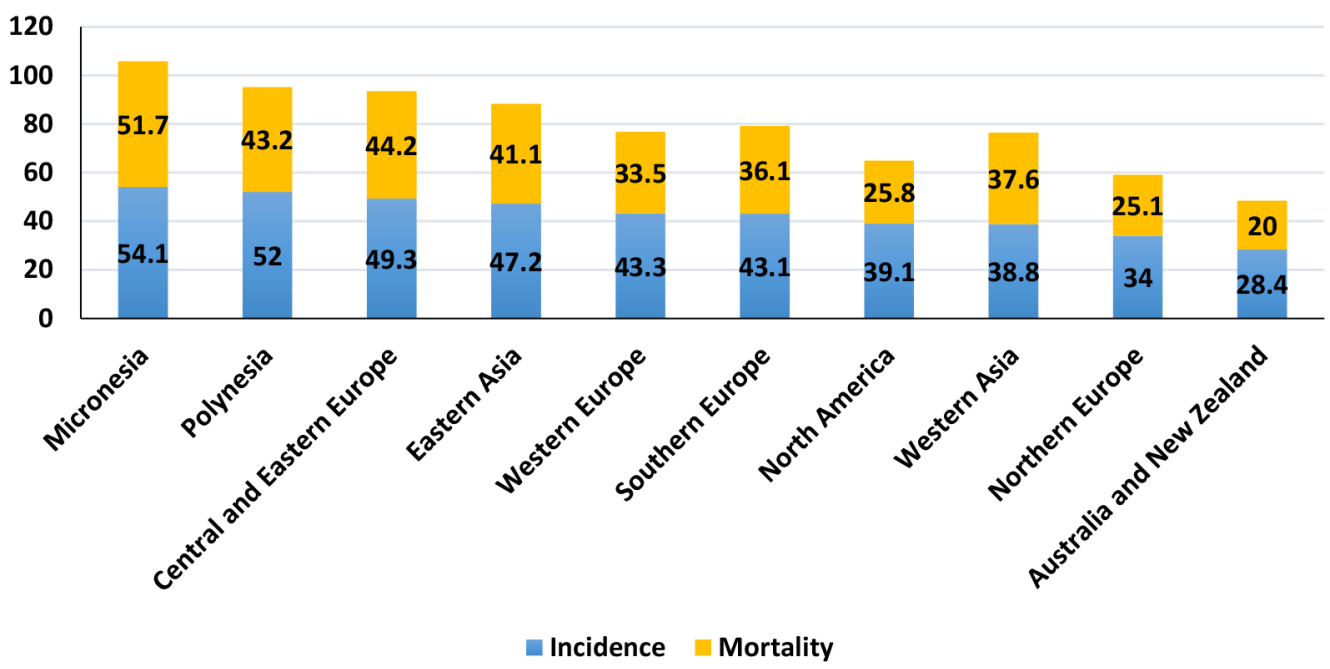

Fig. 6 Bar chart of region-specific incidence and mortality age-standardized rates for lung cancer in 2018. Rates are shown in descending order of the world $(W)$ age-standardized rate.

Source: GLOBOCAN 2018.

\section{Prostate Cancer}

According to 2012 statistics, prostate cancer accounts for $15 \%$ of men's cancers and the second leading cause of cancer death in men (after lung cancer). The average age of diagnosis of prostate cancer is 72 years. It can be said that there is a direct relationship between the age and incidence of prostate cancer. Nearly three-quarters of the cases of prostate cancer diagnosed in the world occur in men over 65 years of age.

Although prostate cancer is widely reported across the world, its prevalence is more common in southern and eastern Asia, Europe, North America, Australia, and New Zealand. 


\begin{tabular}{|c|c|c|c|c|c|c|c|c|c|c|}
\hline \multirow{3}{*}{ Site } & \multicolumn{10}{|c|}{ Age-standardized incidence rates } \\
\hline & \multicolumn{2}{|c|}{ Lung } & \multicolumn{2}{|c|}{ Prostate } & \multicolumn{2}{|c|}{ Colorectum } & \multicolumn{2}{|c|}{ Stomach } & \multicolumn{2}{|c|}{ Liver } \\
\hline & Crud Rate & ASR & Crud Rate & ASR & Crud Rate & ASR & Crud Rate & ASR & Crud Rate & ASR \\
\hline Afghanistan & 4 & 9.4 & 1.6 & 4.5 & 2.6 & 4.7 & 4.6 & 11.5 & 2.1 & 4.9 \\
\hline Albania & 64.7 & 37.8 & 44.5 & 22.9 & 15.2 & 9.6 & 31.2 & 19.1 & 17.9 & 10 \\
\hline Algeria & 15.4 & 17.4 & 12.2 & 13 & 13.7 & 14.8 & 6.5 & 7.1 & 1.5 & 1.6 \\
\hline Angola & 1.6 & 4.2 & 13.4 & 41 & 2.5 & 6 & 1.4 & 3.5 & 2.5 & 5.2 \\
\hline Argentina & 32.5 & 26.3 & 53 & 42.4 & 39 & 31.5 & 11.6 & 9.4 & 6.2 & 5 \\
\hline Armenia & 82.2 & 58.5 & 46.1 & 34.2 & 26.6 & 18.4 & 25.5 & 17 & 19.1 & 13.5 \\
\hline Australia & 58.7 & 29.2 & 148.1 & 85.6 & 78.1 & 41.9 & 12.5 & 6.5 & 14 & 8.6 \\
\hline Austria & 68.5 & 33.2 & 130.4 & 61.6 & 58.9 & 26.3 & 17.1 & 7.4 & 17.5 & 8.2 \\
\hline Azerbaijan & 24.8 & 25.5 & 5.8 & 6.7 & 13.5 & 13.8 & 18.8 & 19.8 & 3.9 & 4.1 \\
\hline Bahamas & 12.8 & 10.4 & 102.3 & 85.8 & 30.7 & 26.4 & 8.2 & 6.9 & 5.1 & 3.9 \\
\hline Bahrain & 5.8 & 16.3 & 3.9 & 10.8 & 7.3 & 14.2 & 1.6 & 3.5 & 2 & 3.9 \\
\hline Bangladesh & 11 & 14.1 & 2.7 & 3.4 & 3.8 & 4.3 & 5.7 & 7 & 2.8 & 3.3 \\
\hline Barbados & 22.6 & 13.7 & 220.4 & 129.3 & 86.1 & 50.3 & 13.1 & 7.2 & 5.1 & 3 \\
\hline Belarus & 82.5 & 54.5 & 76.6 & 49.4 & 63.7 & 41.6 & 38.2 & 25.1 & 7.2 & 4.9 \\
\hline Belgium & 109.4 & 52.2 & 132.6 & 65.5 & 92.7 & 43.8 & 17.8 & 8.4 & 11.2 & 5.9 \\
\hline Belize & 10 & 15.7 & 36.3 & 55.9 & 5.8 & 9.2 & 6.3 & 8.8 & 7.4 & 12.2 \\
\hline Benin & 0.54 & 0.94 & 22.9 & 55.7 & 4.8 & 9.5 & 4.8 & 10.6 & 4.1 & 9.8 \\
\hline Bhutan & 5.5 & 7.1 & 0.69 & 1 & 5.3 & 6 & 18.9 & 24.2 & 6.5 & 7.8 \\
\hline Bolivia, Plurinational State of & 10 & 10.3 & 29 & 34.2 & 5.5 & 6.7 & 8.3 & 9.3 & 5.4 & 5.6 \\
\hline Bosnia and Herzegovina & 111.9 & 62.4 & 54.4 & 26.3 & 60.8 & 33 & 25.9 & 14 & 17.1 & 9.1 \\
\hline Botswana & 3.5 & 6.4 & 6.2 & 13.7 & 3 & 4.9 & 0.61 & 1 & 3.5 & 6.4 \\
\hline Brazil & 18.5 & 16.4 & 82 & 74 & 23.9 & 21.1 & 11.9 & 10.6 & 7.1 & 6.3 \\
\hline Brunei & 25.1 & 34.4 & 15.2 & 22.5 & 37.6 & 43.4 & 13.4 & 17.9 & 14.3 & 17 \\
\hline Bulgaria & 98.2 & 50.1 & 124.4 & 53.6 & 79.6 & 38.3 & 25 & 12 & 11 & 5.7 \\
\hline Burkina Faso & 1.7 & 4.6 & 7 & 26.5 & 2.5 & 5.5 & 3.7 & 9.2 & 8 & 19.2 \\
\hline Burundi & 1.1 & 2.6 & 13.7 & 35.5 & 3.5 & 7.7 & 1.9 & 4.6 & 4.9 & 9 \\
\hline Cabo Verde & 9.4 & 16.3 & 33 & 51.4 & 3.3 & 7.3 & 17.4 & 25.1 & 8.7 & 11.3 \\
\hline Cambodia & 12.8 & 21.6 & 2.4 & 4.5 & 8 & 13 & 3.1 & 5 & 21.3 & 34.6 \\
\hline Cameroon & 1.4 & 3 & 17.9 & 41.6 & 3.6 & 7.7 & 1.6 & 3.3 & 5.9 & 9.3 \\
\hline Canada & 68.1 & 31.1 & 116.8 & 58.2 & 71.1 & 35.2 & 10.9 & 5.2 & 14.2 & 7.6 \\
\hline Central African Republic & 1.5 & 3 & 14.1 & 30.2 & 3.3 & 6.5 & 1.7 & 3.3 & 4.5 & 7.5 \\
\hline Chad & 0.81 & 2 & 7.5 & 22 & 2.8 & 6.6 & 1.4 & 3.5 & 3.6 & 7.8 \\
\hline Chile & 24 & 17 & 72.9 & 51.2 & 33.3 & 23.9 & 38 & 26.9 & 9.7 & 6.9 \\
\hline China & 70.8 & 47.8 & 13.6 & 9.1 & 41.5 & 28.1 & 43.6 & 29.5 & 40 & 27.6 \\
\hline Colombia & 13.4 & 12.7 & 52.3 & 49.8 & 18.1 & 16.9 & 18.8 & 17.6 & 4.9 & 4.6 \\
\hline Comoros & 0.24 & 0.38 & 12.6 & 30.3 & 1.9 & 4.1 & 1.2 & 2.5 & 4.3 & 7.6 \\
\hline Congo, Democratic Republic of & 1.5 & 3.9 & 13.6 & 35.1 & 3.9 & 9 & 3.8 & 7.3 & 5.8 & 11.5 \\
\hline Congo, Republic of & 1.6 & 3.4 & 18.7 & 40.7 & 3 & 5.7 & 1.9 & 3.7 & 5.4 & 8.6 \\
\hline
\end{tabular}




\begin{tabular}{|c|c|c|c|c|c|c|c|c|c|c|}
\hline \multirow{3}{*}{ Site } & \multicolumn{10}{|c|}{ Age-standardized incidence rates } \\
\hline & \multicolumn{2}{|c|}{ Lung } & \multicolumn{2}{|c|}{ Prostate } & \multicolumn{2}{|c|}{ Colorectum } & \multicolumn{2}{|c|}{ Stomach } & \multicolumn{2}{|c|}{ Liver } \\
\hline & Crud Rate & ASR & Crud Rate & ASR & Crud Rate & ASR & Crud Rate & ASR & Crud Rate & ASR \\
\hline Costa Rica & 12.5 & 9.5 & 73.9 & 57.7 & 22.6 & 17.6 & 22.4 & 17.2 & 10 & 7.9 \\
\hline Croatia & 105.7 & 50.9 & 116.8 & 54.5 & 97.2 & 45.9 & 25.1 & 11.8 & 20.4 & 9.9 \\
\hline Cuba & 72.2 & 38.7 & 91.7 & 48.6 & 31.5 & 16.1 & 12.9 & 6.9 & 8.7 & 4.7 \\
\hline Cyprus & 66.4 & 41 & 126.2 & 73.9 & 56.8 & 34.4 & 11.3 & 7.1 & 8.6 & 5 \\
\hline Czech Republic & 80.5 & 38.5 & 176.5 & 88 & 86.9 & 42.5 & 15.4 & 7.4 & 13 & 6.1 \\
\hline Côte d'Ivoire & 1.6 & 3.4 & 19.7 & 43.7 & 2.6 & 4.9 & 1.6 & 3.1 & 5.6 & 9.7 \\
\hline Denmark & 84.7 & 37.3 & 163 & 75.9 & 103.6 & 45.9 & 12.9 & 6 & 14.9 & 7.3 \\
\hline Djibouti & 2.1 & 3 & 4.5 & 7.6 & 4.1 & 5.8 & 1.8 & 2.7 & 2.3 & 3.1 \\
\hline Dominican Republic & 14.2 & 14.6 & 58.1 & 60.1 & 13.5 & 13.9 & 7.7 & 8 & 7.4 & 7.6 \\
\hline Ecuador & 7.2 & 7 & 39.4 & 38.8 & 10.7 & 10.8 & 16.2 & 15.7 & 5.7 & 5.7 \\
\hline Egypt & 8.5 & 11.6 & 6.2 & 9.5 & 5.3 & 6.6 & 2.4 & 3.1 & 36.8 & 49 \\
\hline El Salvador & 7.1 & 6.4 & 47.6 & 43 & 8.9 & 8.9 & 13.1 & 11.9 & 6.9 & 6.4 \\
\hline Equatorial Guinea & 2.7 & 6.4 & 13.3 & 35.9 & 3.3 & 6.4 & 1.4 & 3.1 & 4.7 & 6 \\
\hline Eritrea & 1.6 & 3.3 & 3.6 & 7.7 & 3.7 & 7 & 1.5 & 3 & 1.9 & 3.7 \\
\hline Estonia & 95.1 & 51.4 & 203.8 & 109.9 & 67.9 & 34.8 & 34.1 & 18.7 & 10 & 5.1 \\
\hline Ethiopia & 2 & 3.8 & 3.2 & 6.5 & 4.1 & 7.8 & 1.5 & 3 & 1.4 & 2.6 \\
\hline Fiji & 7.3 & 8 & 42.6 & 45 & 9.3 & 11.3 & 4.3 & 4.5 & 11.5 & 12.3 \\
\hline Finland & 59.7 & 24.8 & 170.4 & 71.6 & 66.8 & 28.7 & 12.4 & 5.3 & 12.7 & 5.2 \\
\hline France & 100.4 & 51.3 & 202.5 & 99 & 80.5 & 36.9 & 15.7 & 7.2 & 26.1 & 13.3 \\
\hline France, Guadeloupe & 26 & 13 & 384.1 & 189.1 & 42.8 & 21.2 & 35.6 & 16.1 & 10.6 & 6.2 \\
\hline France, La Réunion & 51.7 & 34.6 & 95.9 & 63.7 & 43.5 & 29.2 & 21.7 & 14.4 & 13.6 & 9.1 \\
\hline France, Martinique & 30.3 & 12.3 & 329.6 & 158.4 & 63.4 & 29 & 32.6 & 13.1 & 14.3 & 5.9 \\
\hline France, New Caledonia & 78.8 & 59.9 & 120.7 & 93 & 41.9 & 31.7 & 19.2 & 14.4 & 17.7 & 14.3 \\
\hline French Guyana & 22.1 & 27.2 & 73.2 & 92.3 & 22.1 & 26.8 & 15.9 & 19.6 & 12.4 & 15.5 \\
\hline French Polynesia & 64.6 & 55.7 & 83.2 & 73.7 & 18.6 & 15.9 & 11.7 & 10.1 & 15.8 & 13.4 \\
\hline Gabon & 6.9 & 10.9 & 18.1 & 31 & 4.4 & 6.3 & 2.2 & 3.7 & 3.3 & 3.9 \\
\hline The Gambia & 2.7 & 6.2 & 3.1 & 8.4 & 0.84 & 1.7 & 0.93 & 2.3 & 22.9 & 36.5 \\
\hline Gaza Strip and West Bank & 12.1 & 26.7 & 7.5 & 18.1 & 10.2 & 21.1 & 2.9 & 6 & 1.2 & 2.4 \\
\hline Georgia & 55.8 & 35.7 & 33.5 & 20.8 & 17.7 & 11.1 & 20.3 & 12.7 & 12.6 & 8.3 \\
\hline Germany & 97.9 & 41 & 154.5 & 63.2 & 76.5 & 31 & 22.5 & 9.4 & 15.3 & 6.4 \\
\hline Ghana & 0.99 & 1.8 & 14.5 & 32.3 & 4.5 & 9.6 & 3 & 5.7 & 13.7 & 24.4 \\
\hline Greece & 143.4 & 67.8 & 117.8 & 50.5 & 75.8 & 32.3 & 21.1 & 8.9 & 20.4 & 8.6 \\
\hline Guam & 68 & 53.7 & 53.6 & 42.3 & 25 & 20.1 & 9.5 & 7 & 29.8 & 24.5 \\
\hline Guatemala & 2.6 & 3.9 & 25.2 & 39.9 & 4.3 & 6.3 & 9.6 & 14.7 & 10.1 & 15.8 \\
\hline Guinea & 2.1 & 3.7 & 13.8 & 35.3 & 1.2 & 2.1 & 3 & 5.8 & 14.8 & 27.9 \\
\hline Guinea-Bissau & 1.3 & 2.6 & 8.3 & 21.9 & 3.2 & 7.1 & 3.5 & 7.8 & 8.9 & 17.3 \\
\hline Guyana & 3.5 & 5 & 29.6 & 39.3 & 3.8 & 4.4 & 2.5 & 3.7 & 2.5 & 2.7 \\
\hline Haiti & 4 & 6 & 36.4 & 55.4 & 7.4 & 9.9 & 11 & 15.6 & 6.4 & 9.5 \\
\hline
\end{tabular}




\begin{tabular}{|c|c|c|c|c|c|c|c|c|c|c|}
\hline \multirow{3}{*}{ Site } & \multicolumn{10}{|c|}{ Age-standardized incidence rates } \\
\hline & \multicolumn{2}{|c|}{ Lung } & \multicolumn{2}{|c|}{ Prostate } & \multicolumn{2}{|c|}{ Colorectum } & \multicolumn{2}{|c|}{ Stomach } & \multicolumn{2}{|c|}{ Liver } \\
\hline & Crud Rate & ASR & Crud Rate & ASR & Crud Rate & ASR & Crud Rate & ASR & Crud Rate & ASR \\
\hline Honduras & 4.6 & 6.8 & 16.1 & 24.9 & 6.1 & 9 & 8.6 & 12.7 & 6.4 & 9.2 \\
\hline Hungary & 140.3 & 77.4 & 119.5 & 60.2 & 132.6 & 70.6 & 26.6 & 13.8 & 16.7 & 9.2 \\
\hline Iceland & 47.8 & 27.7 & 104.4 & 58.3 & 54.3 & 30.5 & 8.8 & 4.2 & 7.1 & 4.1 \\
\hline India & 6.9 & 7.8 & 3.7 & 4.4 & 5.2 & 5.8 & 5.5 & 6.2 & 2.7 & 3.1 \\
\hline Indonesia & 16.7 & 19.4 & 8.5 & 11.3 & 14.2 & 16.2 & 1.7 & 2.1 & 10.6 & 12.4 \\
\hline Iran, Islamic Republic of & 11.4 & 12.5 & 14.6 & 16.6 & 13.7 & 14.6 & 19.6 & 21.6 & 4.8 & 5.3 \\
\hline Iraq & 7.9 & 17.4 & 2.8 & 6.6 & 3.8 & 7.2 & 2.3 & 4.6 & 1.4 & 3.1 \\
\hline Ireland & 68.6 & 38.8 & 208.8 & 132.5 & 73.5 & 42.4 & 18.2 & 10.3 & 11.5 & 6.8 \\
\hline Israel & 36.9 & 27.8 & 69.7 & 52.2 & 31.8 & 22.8 & 10.3 & 7.3 & 4.8 & 3.5 \\
\hline Italy & 93.1 & 34.5 & 151.6 & 61.3 & 93.1 & 36 & 26.7 & 9.7 & 30.2 & 12.8 \\
\hline Jamaica & 27.3 & 21.1 & 90.8 & 71.9 & 26.4 & 21.3 & 12.6 & 10.2 & 3.8 & 3 \\
\hline Japan & 127.9 & 41.4 & 113.8 & 35.4 & 133.1 & 49.1 & 123.8 & 40.7 & 37.8 & 12.3 \\
\hline Jordan & 19 & 32 & 7.9 & 14.7 & 9.2 & 14.8 & 5 & 8.5 & 2 & 3.4 \\
\hline Kazakhstan & 39.6 & 43.8 & 10.6 & 12.8 & 15.6 & 17.7 & 22.1 & 24.7 & 7.3 & 8.2 \\
\hline Kenya & 1.5 & 3.6 & 11.3 & 30.9 & 4.5 & 10 & 4.1 & 10 & 3 & 6.2 \\
\hline Korea, Democratic Republic of & 54.7 & 48.1 & 3.5 & 3.5 & 26.1 & 22.7 & 26.3 & 23.1 & 30.6 & 25.4 \\
\hline Korea, Republic of & 75.2 & 41.7 & 64.8 & 36.2 & 102.1 & 59.5 & 99.5 & 57.8 & 47.6 & 27.7 \\
\hline Kuwait & 4.6 & 8.7 & 9.2 & 21.6 & 8.1 & 12.9 & 1.8 & 3 & 3.7 & 6.2 \\
\hline Kyrgyzstan & 17.6 & 26.8 & 4.3 & 7.5 & 5.7 & 8.1 & 19.9 & 29.3 & 8.8 & 13 \\
\hline Lao People's Democratic Republic & 18.1 & 29.4 & 2 & 3.8 & 9.4 & 14.9 & 10.4 & 16.2 & 20.5 & 33.4 \\
\hline Latvia & 98 & 51.8 & 155.7 & 80.3 & 82.9 & 42.6 & 35 & 19 & 7.1 & 3.8 \\
\hline Lebanon & 37.1 & 31.3 & 49.2 & 39.3 & 25.4 & 20.8 & 9.9 & 8.2 & 4.4 & 3.6 \\
\hline Lesotho & 3.2 & 6.4 & 12.6 & 25 & 2.5 & 4.4 & 0.27 & 0.7 & 3.7 & 6.6 \\
\hline Liberia & 1.8 & 3.7 & 15.3 & 39.1 & 1.8 & 3.4 & 2.3 & 4.7 & 10.1 & 18.9 \\
\hline Libya & 18.2 & 26.4 & 9.7 & 15.6 & 9.5 & 13 & 3.4 & 4.8 & 3 & 4.4 \\
\hline Lithuania & 95.6 & 52.6 & 117.2 & 70.2 & 67.8 & 35.6 & 36.6 & 19.9 & 12.1 & 6.5 \\
\hline Luxembourg & 72.1 & 40 & 134.1 & 78.8 & 57.3 & 31.4 & 15.8 & 8.1 & 16.2 & 9.1 \\
\hline Madagascar & 0.7 & 1.4 & 13.4 & 31.7 & 2.8 & 5.5 & 1.4 & 2.8 & 4.5 & 8.3 \\
\hline Malawi & 0.87 & 2.4 & 5.5 & 15.3 & 1.8 & 3.9 & 1.2 & 3 & 1.8 & 2.4 \\
\hline Malaysia & 20.7 & 22.5 & 10.9 & 12.4 & 20.2 & 22 & 5.3 & 5.8 & 8.8 & 9.5 \\
\hline Maldives & 12.7 & 18.9 & 5.9 & 10.4 & 11.9 & 15.9 & 0 & 0 & 8.7 & 12.3 \\
\hline Mali & 1.9 & 5.5 & 5.6 & 17.7 & 4 & 9 & 5.2 & 12.4 & 4.6 & 10.2 \\
\hline Malta & 65.9 & 28.2 & 135 & 57.4 & 82.9 & 36 & 19.4 & 8.4 & 6.9 & 3.5 \\
\hline Mauritania & 1.9 & 3.9 & 8.8 & 21.9 & 3.1 & 5.2 & 3.5 & 6.9 & 9.7 & 16.8 \\
\hline Mauritius & 25 & 17.7 & 28.2 & 19.9 & 24.9 & 17.4 & 12.6 & 8.9 & 5.7 & 4 \\
\hline Mexico & 7 & 7.3 & 38.5 & 41.6 & 12 & 12.5 & 6 & 6.2 & 5.3 & 5.5 \\
\hline Mongolia & 23.8 & 36.8 & 2.2 & 3.9 & 4.3 & 6.5 & 33.8 & 47.2 & 84.2 & 117 \\
\hline Montenegro & 101.5 & 62.9 & 63.4 & 34 & 37 & 22.7 & 11.3 & 6.8 & 10.6 & 5.7 \\
\hline Morocco & 32.3 & 31.9 & 22.3 & 22.7 & 12.3 & 12.2 & 6.2 & 6.2 & 1.4 & 1.4 \\
\hline
\end{tabular}




\begin{tabular}{|c|c|c|c|c|c|c|c|c|c|c|}
\hline \multirow{3}{*}{ Site } & \multicolumn{10}{|c|}{ Age-standardized incidence rates } \\
\hline & \multicolumn{2}{|c|}{ Lung } & \multicolumn{2}{|c|}{ Prostate } & \multicolumn{2}{|c|}{ Colorectum } & \multicolumn{2}{|c|}{ Stomach } & \multicolumn{2}{|c|}{ Liver } \\
\hline & Crud Rate & ASR & Crud Rate & ASR & Crud Rate & ASR & Crud Rate & ASR & Crud Rate & ASR \\
\hline Mozambique & 0.86 & 1.9 & 11.1 & 27.1 & 1.9 & 3.7 & 0.78 & 1.8 & 4.5 & 8.8 \\
\hline Myanmar & 16.7 & 19.5 & 3.1 & 4 & 9.9 & 11.1 & 15.5 & 17.6 & 13.4 & 14.6 \\
\hline Namibia & 2.9 & 5.6 & 16.4 & 37.3 & 4 & 7.3 & 1.6 & 3.2 & 2.5 & 4.2 \\
\hline Nepal & 11.8 & 14.8 & 0.87 & 1.1 & 3.6 & 4.4 & 6.2 & 7.8 & 1.2 & 1.5 \\
\hline New Zealand & 47.4 & 24.2 & 163.7 & 90.8 & 77.1 & 40.2 & 10.9 & 5.9 & 16.5 & 10 \\
\hline Nicaragua & 6 & 7.7 & 34.4 & 45.4 & 8.1 & 10.2 & 10.4 & 13.5 & 10.4 & 13.6 \\
\hline Niger & 0.33 & 0.89 & 1.5 & 4.4 & 2.7 & 5.5 & 1.8 & 4 & 5.2 & 11.5 \\
\hline Nigeria & 0.78 & 1.6 & 13.2 & 32.8 & 3.6 & 6.8 & 1.6 & 3.3 & 3.1 & 5.9 \\
\hline Norway & 62.2 & 30.9 & 202.6 & 106.5 & 93.6 & 46.9 & 12.1 & 6 & 9.2 & 5.3 \\
\hline Oman & 3 & 7.1 & 4.5 & 12.7 & 8.2 & 11.7 & 4.9 & 10.9 & 2.8 & 5.8 \\
\hline Pakistan & 7.7 & 11.5 & 4.4 & 6.7 & 3.5 & 4.5 & 2.3 & 3.3 & 2.6 & 3.8 \\
\hline Panama & 11.6 & 10.4 & 65.7 & 60.7 & 20.2 & 18.5 & 16.2 & 14.8 & 5.5 & 4.9 \\
\hline Papua New Guinea & 8.7 & 15.7 & 15.1 & 28.2 & 10.3 & 19.3 & 6.4 & 11.7 & 9.9 & 14 \\
\hline Paraguay & 17 & 19.3 & 38 & 43.4 & 13.3 & 15 & 5 & 5.6 & 3.3 & 3.7 \\
\hline Peru & 9.6 & 9.5 & 46.7 & 47.8 & 13.9 & 14.2 & 19 & 19.3 & 6.8 & 6.8 \\
\hline Philippines & 22.6 & 33.1 & 13.6 & 22.9 & 16.4 & 23.5 & 3.3 & 4.8 & 12.8 & 17.8 \\
\hline Poland & 97.8 & 52.7 & 83.7 & 43.7 & 76.2 & 41.1 & 22.7 & 12.4 & 8.4 & 4.7 \\
\hline Portugal & 82.1 & 38.1 & 135.7 & 59.5 & 125.3 & 54 & 35.2 & 15.5 & 20.2 & 9.2 \\
\hline Puerto Rico & 26.2 & 15 & 159.7 & 101.7 & 55.7 & 34.1 & 9.6 & 5.3 & 14.3 & 8.9 \\
\hline Qatar & 3 & 10.3 & 3.6 & 15.5 & 5 & 13.5 & 1.5 & 5.5 & 1.8 & 5.7 \\
\hline Republic of Moldova & 68.3 & 50.5 & 43 & 32.1 & 64.1 & 47.3 & 24.9 & 18.5 & 27.8 & 20.9 \\
\hline Romania & 90.4 & 50.7 & 63.6 & 30.5 & 68.5 & 36.3 & 24.2 & 12.6 & 23.5 & 12.7 \\
\hline Russian Federation & 72.3 & 48.2 & 59.9 & 39.4 & 49.5 & 32.9 & 30.6 & 20.4 & 8.9 & 6 \\
\hline Rwanda & 1.8 & 4.4 & 11.5 & 29.1 & 5.8 & 12.6 & 7.2 & 11.4 & 7.9 & 14.7 \\
\hline Saint Lucia & 18.2 & 12.9 & 91 & 71.4 & 18.2 & 13.3 & 15.9 & 12.3 & 3.4 & 2.2 \\
\hline Samoa & 33.3 & 44 & 38.2 & 51.5 & 23.5 & 30.6 & 5.9 & 7.7 & 13.7 & 17.3 \\
\hline Sao Tome and Principe & 9.6 & 18.2 & 12.5 & 34 & 2.9 & 9.5 & 7.7 & 11.6 & 6.7 & 14.8 \\
\hline Saudi Arabia & 3.6 & 5.8 & 3.2 & 6.1 & 12.5 & 14.9 & 1.9 & 2.6 & 3.5 & 6.2 \\
\hline Senegal & 1.6 & 3.7 & 12 & 32.2 & 2.8 & 6.3 & 3 & 6.7 & 8.4 & 18 \\
\hline Serbia & 125.4 & 71.6 & 74.3 & 35.4 & 88.2 & 49 & 18.6 & 10.2 & 10.7 & 5.9 \\
\hline Sierra Leone & 1.5 & 3.2 & 10.2 & 29 & 2.7 & 5 & 3.1 & 6.9 & 6.5 & 12.6 \\
\hline Singapore & 75.4 & 41.5 & 117.8 & 64.1 & 69.7 & 38.9 & 19.9 & 10.8 & 34.9 & 19.5 \\
\hline Slovakia & 91.4 & 54.3 & 89.2 & 50.5 & 102.8 & 60.7 & 26.1 & 15.4 & 12.6 & 7.7 \\
\hline Slovenia & 96.7 & 46.6 & 170.1 & 79.3 & 125.8 & 58.9 & 27.4 & 12.5 & 20.9 & 9.7 \\
\hline Solomon Islands & 5.7 & 11 & 13.6 & 24.5 & 3.8 & 7.3 & 1.3 & 2.3 & 10.1 & 15.5 \\
\hline Somalia & 1.7 & 3.7 & 5.1 & 13.1 & 3.9 & 8.4 & 2.1 & 4.8 & 1.7 & 3.6 \\
\hline South Africa & 19.6 & 28.2 & 44.2 & 68 & 12.5 & 18.1 & 4.1 & 5.8 & 5.5 & 7.6 \\
\hline South Sudan & 1.8 & 3.6 & 11.1 & 24.3 & 4 & 7.5 & 2.4 & 5 & 3.8 & 6.5 \\
\hline Spain & 89.8 & 42.1 & 139.4 & 73.1 & 99.9 & 45.2 & 20.9 & 9.2 & 21.9 & 10.9 \\
\hline
\end{tabular}




\begin{tabular}{|c|c|c|c|c|c|c|c|c|c|c|}
\hline \multirow{3}{*}{ Site } & \multicolumn{10}{|c|}{ Age-standardized incidence rates } \\
\hline & \multicolumn{2}{|c|}{ Lung } & \multicolumn{2}{|c|}{ Prostate } & \multicolumn{2}{|c|}{ Colorectum } & \multicolumn{2}{|c|}{ Stomach } & \multicolumn{2}{|c|}{ Liver } \\
\hline & Crud Rate & ASR & Crud Rate & ASR & Crud Rate & ASR & Crud Rate & ASR & Crud Rate & ASR \\
\hline Sri Lanka & 10.6 & 8.3 & 5.3 & 4 & 7.3 & 5.8 & 5.9 & 4.6 & 4.7 & 3.6 \\
\hline Sudan & 1.6 & 2.9 & 4.5 & 9.2 & 3.6 & 6.2 & 1.6 & 3 & 2.8 & 4.8 \\
\hline Suriname & 25.6 & 25.7 & 62.1 & 69.3 & 17.9 & 18.8 & 7 & 7 & 8.8 & 9 \\
\hline Swaziland & 1.6 & 4.3 & 12.2 & 34.2 & 2.4 & 4.6 & 0.3 & 1.1 & 3 & 5 \\
\hline Sweden & 39.4 & 16.9 & 211.6 & 103 & 67.4 & 29.7 & 9.7 & 4.2 & 12.8 & 6.5 \\
\hline Switzerland & 57.4 & 26.6 & 160.1 & 77.4 & 61.3 & 28.4 & 15 & 7.2 & 15.7 & 7.3 \\
\hline Syrian Arab Republic & 16.8 & 26.6 & 12.3 & 20.1 & 9.4 & 14.3 & 4.6 & 7.1 & 2.2 & 3.4 \\
\hline Tajikistan & 4.6 & 7.5 & 1.2 & 2.3 & 3.6 & 4.9 & 11.7 & 20.2 & 3.6 & 6 \\
\hline Tanzania, United Republic of & 0.38 & 0.85 & 14.9 & 36 & 3.4 & 7.4 & 1.8 & 3.8 & 4 & 8.1 \\
\hline Thailand & 45.9 & 29.4 & 19.2 & 12.3 & 26.7 & 17.6 & 6.3 & 4.1 & 48.3 & 32.2 \\
\hline The Netherlands & 76.5 & 34.5 & 149.4 & 68.9 & 100.1 & 45.3 & 14.2 & 6.2 & 7.3 & 3.4 \\
\hline $\begin{array}{l}\text { The former Yugoslav Republic of } \\
\text { Macedonia }\end{array}$ & 87.2 & 55.7 & 67.7 & 39.2 & 49.6 & 30.9 & 24.9 & 15.4 & 11.6 & 7.1 \\
\hline Timor-Leste & 7.6 & 15 & 3.7 & 8 & 4.9 & 9.7 & 2.5 & 5.5 & 4 & 7.3 \\
\hline Togo & 1.4 & 3.1 & 7.8 & 20.4 & 3.8 & 7.2 & 4.2 & 8.7 & 5.2 & 9.7 \\
\hline Trinidad and Tobago & 28.7 & 21.8 & 89.6 & 68.1 & 28.3 & 21.5 & 8.1 & 6.2 & 5.3 & 3.9 \\
\hline Tunisia & 30 & 26.5 & 14.2 & 12.3 & 14.7 & 13 & 6.5 & 5.6 & 2.8 & 2.5 \\
\hline Turkey & 72.8 & 70.6 & 42.9 & 41.7 & 28.6 & 27.4 & 18.4 & 17.6 & 6.9 & 6.6 \\
\hline Turkmenistan & 11.4 & 15.7 & 3.3 & 5.6 & 4.7 & 6.7 & 11.7 & 17.3 & 6.1 & 8.4 \\
\hline Uganda & 1.4 & 4.3 & 9.5 & 34.5 & 3 & 8.3 & 1.7 & 5.5 & 5.3 & 10.1 \\
\hline Ukraine & 66.7 & 41.7 & 54.6 & 32 & 55 & 33.6 & 28.3 & 17.5 & 4.9 & 3.2 \\
\hline United Arab Emirates & 2.1 & 7 & 3.7 & 15.8 & 6.4 & 14.8 & 1.1 & 4.1 & 0.9 & 4.3 \\
\hline United Kingdom & 82.2 & 35.5 & 171.6 & 80.7 & 80.8 & 37.8 & 12.5 & 5.3 & 14.7 & 7 \\
\hline United States of America & 75 & 40.1 & 131.5 & 75.7 & 50 & 28.8 & 10.2 & 5.6 & 16.9 & 10.4 \\
\hline Uruguay & 69.1 & 46.2 & 90.1 & 59.6 & 68.7 & 43.8 & 19.4 & 12.5 & 6.8 & 4.4 \\
\hline Uzbekistan & 10.3 & 14.1 & 2.8 & 4.2 & 5 & 6.5 & 9.9 & 13.8 & 4.9 & 6.8 \\
\hline Vanuatu & 8.4 & 14.4 & 15.4 & 25 & 2.8 & 4.3 & 1.4 & 2.2 & 15.4 & 20.2 \\
\hline Venezuela, Bolivarian Republic of & 18.3 & 19.7 & 46.2 & 51.2 & 13.8 & 14.8 & 8.5 & 9.2 & 4 & 4.3 \\
\hline Viet Nam & 35 & 35.4 & 8.3 & 8.4 & 15.9 & 16 & 23.4 & 23.3 & 41 & 39 \\
\hline Yemen & 1.8 & 3.9 & 0.81 & 1.8 & 4.4 & 9.5 & 3.7 & 8.6 & 2.8 & 6.3 \\
\hline Zambia & 1.3 & 4 & 14.1 & 45.6 & 1.9 & 4.9 & 1.3 & 3.4 & 1.2 & 2.8 \\
\hline Zimbabwe & 2.2 & 5.8 & 2018 & 43.5 & 4.6 & 11.6 & 2.6 & 6.9 & 3.9 & 8.7 \\
\hline
\end{tabular}

However, more than $50 \%$ of deaths from prostate cancer have occurred in more developed countries. ${ }^{6-5}$ Based on cancer record results in 2018, prostate cancer has the highest incidence in men with $1,276,106$ cases $(29.3 \%$ in 100,000$)$ after lung cancer $(31.5 \%$ in 100,000$)$. With a mortality of 6.7 per 100,000 , it is the sixth leading cause of death from cancer in men. Results showed the highest incidence of lung cancer was found in Australia and New Zealand (86.4\% in 100,000), Northern Europe $(85.7 \%$ in 100,000$)$, and Western Europe
(75.8\% in 100,000). The highest mortality rates related to the highest incidence of prostate cancer in the world was in France, Guadeloupe (189.1 in 100,000), France, Martinique (158.1 in 100,000), and Ireland (13.13 in 100,000) (Table 1, Fig. 9), respectively.

According to the results of 2018, the highest mortality rate for prostate cancer was in Barbados (48 per 100,000), Jamaica (41.7 per 100,000$)$, and Benin (36.3 per 100,000$)$, respectively (Table 2). 


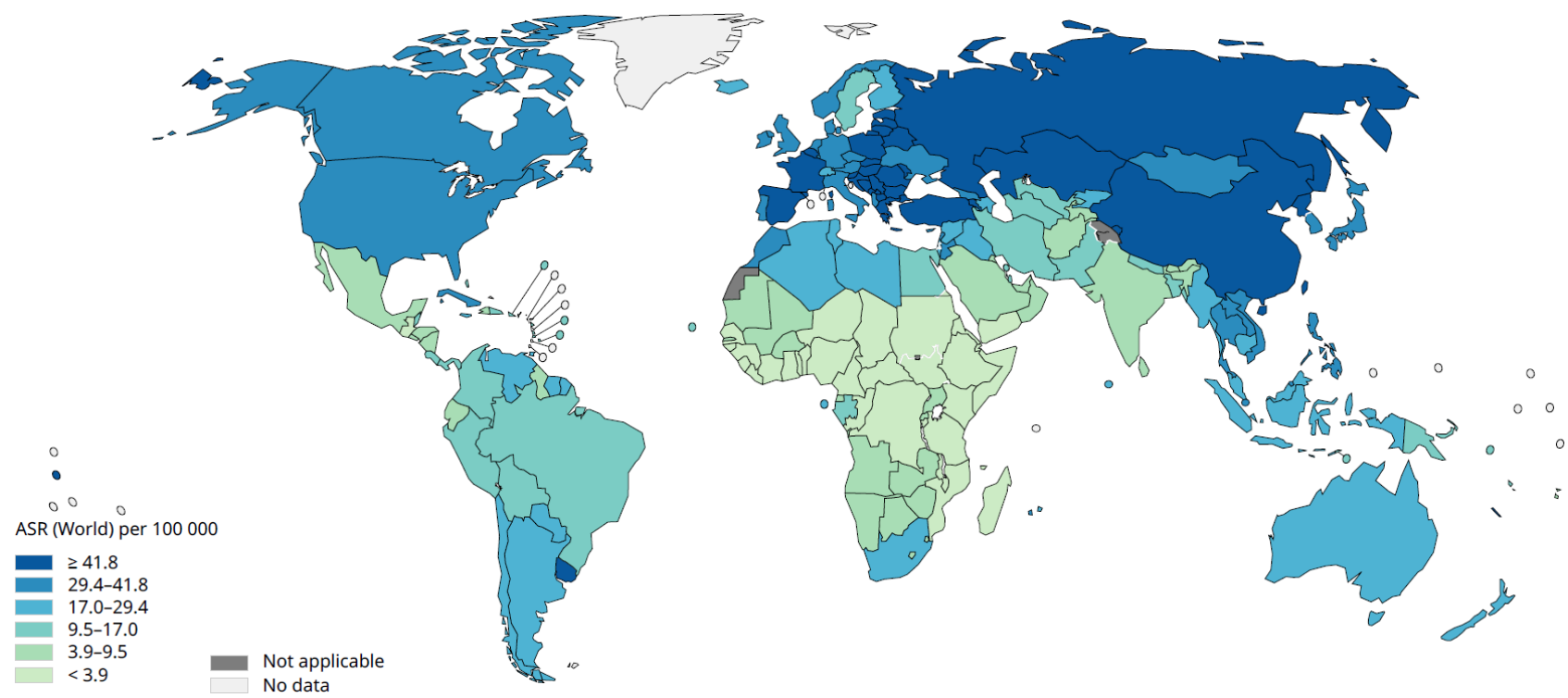

Fig. 7 Global map presenting age-standardized incidence rates by world countries for lung cancer in males in 2018.

Source: GLOBOCAN 2018.

\begin{tabular}{|c|c|c|c|c|c|c|c|c|c|c|}
\hline \multirow{3}{*}{ Site } & \multicolumn{10}{|c|}{ Age-standardized mortality rates } \\
\hline & \multicolumn{2}{|c|}{ Lung } & \multicolumn{2}{|c|}{ Prostate } & \multicolumn{2}{|c|}{ Colorectum } & \multicolumn{2}{|c|}{ Stomach } & \multicolumn{2}{|c|}{ Liver } \\
\hline & Crud Rate & ASR & Crud Rate & ASR & Crud Rate & ASR & Crud Rate & ASR & Crud Rate & ASR \\
\hline Afghanistan & 4 & 9.5 & 1.3 & 3.8 & 2.3 & 4.2 & 4.5 & 11.4 & 2 & 4.7 \\
\hline Albania & 58 & 33.1 & 20.3 & 9.6 & 7.8 & 4.5 & 24.4 & 14.3 & 17.5 & 9.6 \\
\hline Algeria & 15.6 & 17.3 & 4.9 & 4.5 & 7.9 & 8.5 & 5.9 & 6.5 & 1.4 & 1.6 \\
\hline Angola & 1.6 & 4.2 & 7.6 & 25.2 & 1.7 & 4.3 & 1.3 & 3.2 & 2.3 & 4.8 \\
\hline Argentina & 30.8 & 24.7 & 18.2 & 12.3 & 21.7 & 16.6 & 9.4 & 7.5 & 5.5 & 4.4 \\
\hline Armenia & 76.1 & 54.5 & 26.5 & 16.8 & 18.3 & 12.6 & 24.1 & 16.1 & 19 & 13.5 \\
\hline Australia & 41.7 & 20.3 & 26.7 & 10 & 26.2 & 12.8 & 6.2 & 3.1 & 13.1 & 7.2 \\
\hline Austria & 61.7 & 28.1 & 29.4 & 9.5 & 30.7 & 12 & 10.4 & 4.3 & 16.2 & 7.2 \\
\hline Azerbaijan & 23.4 & 24 & 3.1 & 3.7 & 9.7 & 10.2 & 17.4 & 18.3 & 3.7 & 3.9 \\
\hline Bahamas & 12.3 & 10.1 & 40.9 & 34.1 & 14.3 & 12.3 & 7.2 & 6 & 5.1 & 3.9 \\
\hline Bahrain & 5.5 & 15.2 & 1.4 & 4.3 & 4 & 8.9 & 1.5 & 3.5 & 1.8 & 3.7 \\
\hline Bangladesh & 10.6 & 13.5 & 2 & 2.4 & 3.1 & 3.5 & 5.4 & 6.6 & 2.5 & 3.1 \\
\hline Barbados & 19.7 & 11.8 & 101.4 & 48 & 38.7 & 21.4 & 10.9 & 5.8 & 5.1 & 3 \\
\hline Belarus & 58.3 & 38.3 & 21.6 & 13.6 & 29.9 & 19.2 & 23.9 & 15.6 & 5.7 & 3.8 \\
\hline Belgium & 82.3 & 37.6 & 26.8 & 8.7 & 29.4 & 11.9 & 8.8 & 3.8 & 11.1 & 5.1 \\
\hline Belize & 10 & 15.7 & 16.3 & 27.6 & 3.7 & 6.2 & 5.8 & 8.1 & 7.4 & 12.2 \\
\hline Benin & 0.54 & 0.94 & 14.5 & 36.3 & 3.3 & 6.8 & 4.9 & 10.8 & 4.1 & 9.9 \\
\hline Bhutan & 5.1 & 6.6 & 0.69 & 1 & 4.4 & 5.5 & 18.2 & 23.4 & 6.2 & 7.6 \\
\hline Bolivia, Plurinational State of & 9.7 & 9.9 & 8.9 & 9.1 & 3.5 & 4.1 & 7.3 & 8 & 5.3 & 5.5 \\
\hline Bosnia and Herzegovina & 100.6 & 53.8 & 27.1 & 11.5 & 36.5 & 18.2 & 20.7 & 10.6 & 16 & 7.9 \\
\hline Botswana & 3.4 & 6.4 & 3.9 & 9.3 & 1.7 & 3.2 & 0.52 & 0.93 & 3.4 & 6.3 \\
\hline Brazil & 17.4 & 15.4 & 16.1 & 13.6 & 11.6 & 10.1 & 9.6 & 8.5 & 6.7 & 5.9 \\
\hline
\end{tabular}




\begin{tabular}{|c|c|c|c|c|c|c|c|c|c|c|}
\hline \multirow{3}{*}{ Site } & \multicolumn{10}{|c|}{ Age-standardized mortality rates } \\
\hline & \multicolumn{2}{|c|}{ Lung } & \multicolumn{2}{|c|}{ Prostate } & \multicolumn{2}{|c|}{ Colorectum } & \multicolumn{2}{|c|}{ Stomach } & \multicolumn{2}{|c|}{ Liver } \\
\hline & Crud Rate & ASR & Crud Rate & ASR & Crud Rate & ASR & Crud Rate & ASR & Crud Rate & ASR \\
\hline Brunei & 18.8 & 26.3 & 3.1 & 5.2 & 14.3 & 18.5 & 8.5 & 12.4 & 11.6 & 14 \\
\hline Bulgaria & 87.3 & 44 & 32.7 & 12.5 & 48 & 21.3 & 19.7 & 9.1 & 11.1 & 5.4 \\
\hline Burkina Faso & 1.7 & 4.6 & 5.1 & 20.6 & 2 & 4.8 & 3.7 & 9.2 & 7.8 & 18.7 \\
\hline Burundi & 1 & 2.5 & 8.3 & 23.4 & 3 & 7 & 1.8 & 4.5 & 5.2 & 9.7 \\
\hline Cabo Verde & 9.4 & 16.3 & 21 & 30.1 & 2.2 & 4.2 & 16.3 & 23.4 & 9.4 & 11.8 \\
\hline Cambodia & 12.4 & 21.1 & 1.6 & 3.3 & 5.2 & 8.8 & 2.6 & 4.4 & 21.4 & 34.7 \\
\hline Cameroon & 1.5 & 3 & 9.4 & 22.4 & 2.6 & 5.8 & 1.6 & 3.3 & 5.5 & 9.2 \\
\hline Canada & 57.9 & 25.4 & 21.7 & 7.8 & 27.7 & 12.2 & 6.2 & 2.8 & 12.6 & 6 \\
\hline Central African Republic & 1.5 & 3 & 10 & 21.3 & 3.1 & 6.3 & 1.6 & 3.3 & 3.9 & 6.9 \\
\hline Chad & 0.75 & 2 & 4.3 & 13.5 & 2.2 & 5.6 & 1.4 & 3.4 & 3.3 & 7.5 \\
\hline Chile & 22.8 & 16.1 & 25.2 & 15.8 & 17.3 & 11.9 & 26 & 17.9 & 8.7 & 6.1 \\
\hline China & 64.4 & 43.4 & 7.1 & 4.7 & 19.4 & 13.1 & 37 & 25 & 37.3 & 25.6 \\
\hline Colombia & 12.2 & 11.5 & 13 & 12 & 9.1 & 8.4 & 14.2 & 13.2 & 4.8 & 4.5 \\
\hline Comoros & 0.24 & 0.38 & 7.6 & 19.5 & 1.9 & 4.1 & 1.2 & 2.5 & 4.3 & 7.8 \\
\hline Congo, Democratic Republic of & 1.4 & 3.7 & 9 & 23.8 & 3.1 & 7.5 & 3.3 & 6.8 & 5.9 & 11.9 \\
\hline Congo, Republic of & 1.6 & 3.4 & 11.1 & 23.8 & 2.1 & 4.1 & 1.9 & 3.6 & 4.8 & 8 \\
\hline Costa Rica & 10.4 & 7.7 & 18.5 & 12.2 & 12.8 & 9.5 & 18.7 & 13.9 & 9.6 & 7.2 \\
\hline Croatia & 104.4 & 50.1 & 42.7 & 15.5 & 64.1 & 27.4 & 22 & 9.9 & 18.1 & 8.4 \\
\hline Cuba & 64.4 & 33.6 & 54.3 & 22.3 & 21.4 & 10.2 & 9.8 & 5 & 7.8 & 4.1 \\
\hline Cyprus & 69.3 & 42.2 & 31.8 & 15.1 & 25.9 & 14.6 & 10.1 & 6.1 & 10.8 & 6.3 \\
\hline Czech Republic & 65.6 & 30.8 & 29.2 & 11.9 & 37.8 & 17.4 & 10.7 & 5.1 & 10.8 & 4.8 \\
\hline Côte d'Ivoire & 1.6 & 3.4 & 12.2 & 28.9 & 2.1 & 3.9 & 1.5 & 2.9 & 5.6 & 9.8 \\
\hline Denmark & 74 & 30.3 & 47.3 & 15.8 & 35 & 13.7 & 8.8 & 3.9 & 12.9 & 5.9 \\
\hline Djibouti & 2.1 & 3 & 3.1 & 5.3 & 3.7 & 5.4 & 1.6 & 2.5 & 2.1 & 2.9 \\
\hline Dominican Republic & 13.6 & 13.7 & 32.4 & 28 & 7.9 & 7.9 & 6.1 & 6.2 & 6.5 & 6.7 \\
\hline Ecuador & 6.9 & 6.6 & 15.9 & 13.9 & 6 & 5.9 & 13.4 & 12.8 & 5.6 & 5.5 \\
\hline Egypt & 8 & 11.1 & 2.5 & 4.3 & 3 & 3.8 & 1.9 & 2.5 & 36.2 & 48.4 \\
\hline El Salvador & 6.8 & 6.1 & 17.5 & 12.8 & 4.8 & 4.5 & 11 & 9.7 & 6.7 & 6.2 \\
\hline Equatorial Guinea & 2.6 & 6.3 & 7 & 19.4 & 2.5 & 5.8 & 1.2 & 2.9 & 4.5 & 6.5 \\
\hline Eritrea & 1.6 & 3.3 & 2.5 & 5.4 & 3 & 5.9 & 1.4 & 2.9 & 1.9 & 3.7 \\
\hline Estonia & 86.6 & 45.1 & 50.9 & 21.8 & 38.2 & 17.8 & 24.2 & 12.7 & 9.3 & 4.8 \\
\hline Ethiopia & 2 & 3.8 & 2.2 & 4.6 & 3.2 & 6.3 & 1.5 & 3 & 1.4 & 2.7 \\
\hline Fiji & 6.9 & 7.7 & 10.2 & 12.8 & 5.8 & 7.9 & 3.7 & 3.9 & 11.5 & 12.2 \\
\hline Finland & 51.5 & 20.8 & 33.4 & 10.8 & 27.6 & 10.8 & 9 & 3.7 & 13.8 & 5.5 \\
\hline France & 81.5 & 38.9 & 28.1 & 8.1 & 33.7 & 13.1 & 10.9 & 4.7 & 23 & 10.4 \\
\hline France, Guadeloupe & 23.6 & 11.8 & 63 & 20 & 29.3 & 13.1 & 23.1 & 10 & 9.6 & 4.2 \\
\hline France, La Réunion & 54 & 35.9 & 23.9 & 14 & 16.8 & 10.9 & 15 & 9.7 & 14 & 9.3 \\
\hline France, Martinique & 30.3 & 12.3 & 65.1 & 18.7 & 32 & 13.3 & 26.3 & 10.3 & 12.6 & 5.1 \\
\hline
\end{tabular}




\begin{tabular}{|c|c|c|c|c|c|c|c|c|c|c|}
\hline \multirow{3}{*}{ Site } & \multicolumn{10}{|c|}{ Age-standardized mortality rates } \\
\hline & \multicolumn{2}{|c|}{ Lung } & \multicolumn{2}{|c|}{ Prostate } & \multicolumn{2}{|c|}{ Colorectum } & \multicolumn{2}{|c|}{ Stomach } & \multicolumn{2}{|c|}{ Liver } \\
\hline & Crud Rate & ASR & Crud Rate & ASR & Crud Rate & ASR & Crud Rate & ASR & Crud Rate & ASR \\
\hline France, New Caledonia & 63.9 & 47.7 & 17.7 & 12.8 & 16.3 & 13 & 12.8 & 9.8 & 13.5 & 10.6 \\
\hline French Guyana & 15.2 & 19.2 & 11.7 & 16.1 & 5.5 & 7.3 & 6.9 & 9 & 7.6 & 9.5 \\
\hline French Polynesia & 57.7 & 49.6 & 24.1 & 21.4 & 6.2 & 5.6 & 8.2 & 7.1 & 15.8 & 13.4 \\
\hline Gabon & 6.8 & 10.8 & 9.8 & 15.5 & 2.4 & 3.8 & 2 & 3.4 & 3.1 & 3.9 \\
\hline The Gambia & 2.7 & 6.3 & 1.7 & 4.9 & 0.65 & 1.5 & 0.93 & 2.3 & 24.9 & 41.1 \\
\hline Gaza Strip and West Bank & 11.5 & 25.5 & 2.4 & 6.4 & 6.2 & 13.3 & 2.8 & 5.8 & 1.2 & 2.4 \\
\hline Georgia & 51.3 & 33.4 & 15.1 & 8.5 & 12.1 & 7.5 & 16.2 & 10.3 & 12.2 & 8 \\
\hline Germany & 79.3 & 31.8 & 39.1 & 11.3 & 36.8 & 13 & 14 & 5.3 & 14.6 & 5.7 \\
\hline Ghana & 0.88 & 1.7 & 7.5 & 18.1 & 3.2 & 7.1 & 3 & 5.8 & 13.6 & 24.3 \\
\hline Greece & 122 & 54.6 & 33.8 & 8.8 & 36.2 & 12.7 & 15.6 & 6 & 17.9 & 6.7 \\
\hline Guam & 65.6 & 51.5 & 21.5 & 16.3 & 14.3 & 11.6 & 4.8 & 3.2 & 23.8 & 19.2 \\
\hline Guatemala & 2.5 & 3.8 & 9.7 & 13.2 & 2.4 & 3.5 & 8.2 & 12.3 & 9.8 & 15.4 \\
\hline Guinea & 1.8 & 3.2 & 10.5 & 28.2 & 0.99 & 1.9 & 2.9 & 5.8 & 13.2 & 25 \\
\hline Guinea-Bissau & 1.3 & 2.6 & 5.2 & 15 & 3 & 6.9 & 3.4 & 7.7 & 9.1 & 17.5 \\
\hline Guyana & 3.5 & 5 & 10.6 & 15.4 & 3 & 3.5 & 2.8 & 4 & 2.5 & 2.8 \\
\hline Haiti & 3.7 & 5.5 & 23.6 & 36.2 & 4.9 & 6.7 & 9.4 & 13.6 & 6.3 & 9.1 \\
\hline Honduras & 4.3 & 6.4 & 8 & 11.6 & 3.7 & 4.9 & 6.9 & 10.4 & 5.9 & 8.2 \\
\hline Hungary & 116.2 & 62.9 & 26.6 & 11.8 & 62.2 & 31.2 & 19.4 & 9.9 & 13.6 & 7.3 \\
\hline Iceland & 40.7 & 20.2 & 37.2 & 14.8 & 27.1 & 13.4 & 5.3 & 2.6 & 10 & 5.1 \\
\hline India & 6.5 & 7.3 & 2.4 & 2.9 & 4.1 & 4.6 & 5 & 5.7 & 2.5 & 2.8 \\
\hline Indonesia & 14.7 & 17.4 & 3.7 & 5.7 & 7.7 & 9.3 & 1.5 & 1.9 & 10.5 & 12.3 \\
\hline Iran, Islamic Republic of & 10.4 & 11.3 & 7.4 & 8.3 & 5.8 & 6.3 & 14.4 & 15.9 & 4.8 & 5.2 \\
\hline Iraq & 7.7 & 17 & 0.8 & 2 & 2.2 & 4.4 & 2.2 & 4.4 & 1.4 & 3.1 \\
\hline Ireland & 46.2 & 25.3 & 24.5 & 11.4 & 28.8 & 15.2 & 8.2 & 4.4 & 10.3 & 5.7 \\
\hline Israel & 36.2 & 26.7 & 10.3 & 5.6 & 17.1 & 11 & 7.4 & 5.2 & 5.6 & 4.1 \\
\hline Italy & 83.1 & 28.7 & 24.5 & 6 & 39.7 & 12.8 & 19.5 & 6.5 & 24.6 & 9.2 \\
\hline Jamaica & 25.6 & 19.4 & 64.4 & 41.7 & 12.9 & 9.8 & 9.9 & 7.8 & 3.7 & 2.9 \\
\hline Japan & 92.3 & 26.5 & 20 & 4.4 & 48.7 & 15.2 & 50.2 & 14.3 & 29.9 & 8.6 \\
\hline Jordan & 17 & 29.3 & 2.5 & 4.9 & 5.1 & 8.5 & 4.7 & 8 & 1.9 & 3.3 \\
\hline Kazakhstan & 35.5 & 39.5 & 5.6 & 7 & 11.5 & 13.3 & 19.6 & 22.1 & 6.8 & 7.6 \\
\hline Kenya & 1.5 & 3.6 & 6.6 & 18.3 & 2.9 & 6.8 & 3.9 & 9.8 & 3 & 6.2 \\
\hline Korea, Democratic Republic of & 48.8 & 44.3 & 1.7 & 1.8 & 11.3 & 10.4 & 18 & 16.3 & 27.9 & 23.5 \\
\hline Korea, Republic of & 58.1 & 31.4 & 8.8 & 4.7 & 21.3 & 11.8 & 17.9 & 10 & 34.5 & 19.5 \\
\hline Kuwait & 4.2 & 8 & 2.2 & 7.5 & 4 & 7.4 & 1.7 & 2.9 & 3.4 & 6 \\
\hline Kyrgyzstan & 16.1 & 24.3 & 2.7 & 4.7 & 4.5 & 6.4 & 18 & 26.6 & 8.3 & 12.2 \\
\hline $\begin{array}{l}\text { Lao People's Democratic } \\
\text { Republic }\end{array}$ & 17.5 & 28.8 & 1 & 2.1 & 5.7 & 9.4 & 9 & 14.4 & 20.5 & 33.4 \\
\hline Latvia & 77.3 & 41.2 & 48.4 & 21 & 36.2 & 17.3 & 30.7 & 16 & 9.8 & 5.3 \\
\hline Lebanon & 34.4 & 28.9 & 16.7 & 11.9 & 14.8 & 11.7 & 8.8 & 7.2 & 4.2 & 3.5 \\
\hline
\end{tabular}




\begin{tabular}{|c|c|c|c|c|c|c|c|c|c|c|}
\hline \multirow{3}{*}{ Site } & \multicolumn{10}{|c|}{ Age-standardized mortality rates } \\
\hline & \multicolumn{2}{|c|}{ Lung } & \multicolumn{2}{|c|}{ Prostate } & \multicolumn{2}{|c|}{ Colorectum } & \multicolumn{2}{|c|}{ Stomach } & \multicolumn{2}{|c|}{ Liver } \\
\hline & Crud Rate & ASR & Crud Rate & ASR & Crud Rate & ASR & Crud Rate & ASR & Crud Rate & ASR \\
\hline Lesotho & 3.2 & 6.4 & 9.2 & 17.7 & 1.9 & 3.6 & 0.27 & 0.7 & 3.7 & 6.6 \\
\hline Liberia & 1.8 & 3.7 & 10.9 & 29 & 1.5 & 3 & 2.3 & 4.7 & 10.4 & 19.2 \\
\hline Libya & 15.4 & 23 & 2.9 & 4.6 & 5.9 & 8.5 & 2.4 & 3.5 & 2.2 & 3.4 \\
\hline Lithuania & 82.8 & 45.1 & 41.5 & 18.1 & 40.4 & 19.6 & 33.2 & 17.4 & 10.3 & 5.5 \\
\hline Luxembourg & 51.9 & 28.4 & 18.9 & 8.2 & 24.3 & 12.3 & 8.1 & 4 & 12.1 & 6.5 \\
\hline Madagascar & 0.65 & 1.3 & 7.4 & 18.6 & 2 & 4.2 & 1.2 & 2.5 & 4.5 & 8.5 \\
\hline Malawi & 0.81 & 2.3 & 3.7 & 10.4 & 1.3 & 2.9 & 1.2 & 3 & 1.9 & 2.9 \\
\hline Malaysia & 18.1 & 19.9 & 4.8 & 5.6 & 11.3 & 12.5 & 4.3 & 4.7 & 8.9 & 9.6 \\
\hline Maldives & 10.7 & 16.6 & 4.3 & 6.6 & 6.7 & 10.5 & 0.4 & 0.36 & 8.3 & 12.1 \\
\hline Mali & 1.9 & 5.5 & 3.8 & 12.2 & 3 & 7.3 & 5 & 12 & 4.2 & 9.7 \\
\hline Malta & 69.6 & 29.6 & 19.8 & 7.7 & 32.3 & 13.2 & 14.7 & 6.4 & 7.4 & 3.6 \\
\hline Mauritania & 1.9 & 3.9 & 5.2 & 13.9 & 2.1 & 3.8 & 3.5 & 6.8 & 9.8 & 16.9 \\
\hline Mauritius & 22.5 & 15.7 & 13.9 & 9.8 & 14.7 & 10.3 & 8.6 & 6 & 4.3 & 2.9 \\
\hline Mexico & 6.3 & 6.5 & 10.6 & 10 & 5.7 & 5.9 & 4.9 & 5 & 5.2 & 5.3 \\
\hline Mongolia & 20.4 & 32.4 & 1.1 & 2.1 & 2.7 & 4 & 25.3 & 36.7 & 69.1 & 98.4 \\
\hline Montenegro & 86.3 & 51.9 & 25.1 & 12.2 & 20 & 11.2 & 9.7 & 5.7 & 10.6 & 5.7 \\
\hline Morocco & 32.4 & 32.1 & 10.4 & 10.9 & 7.7 & 7.7 & 6.1 & 6.1 & 1.3 & 1.3 \\
\hline Mozambique & 0.86 & 2 & 6.8 & 16.9 & 1.5 & 3.2 & 0.76 & 1.8 & 4.7 & 9.4 \\
\hline Myanmar & 16.4 & 19.3 & 1.4 & 2 & 6.1 & 7.1 & 12.7 & 14.8 & 13.6 & 14.7 \\
\hline Namibia & 2.8 & 5.5 & 7.9 & 19.4 & 2.3 & 4.3 & 1.5 & 3.1 & 2.5 & 4.2 \\
\hline Nepal & 11.1 & 14.1 & 0.63 & 0.79 & 3.1 & 3.9 & 5.8 & 7.3 & 1.1 & 1.3 \\
\hline New Zealand & 37.4 & 18.5 & 28.7 & 11.6 & 28.1 & 13.4 & 8.3 & 4.2 & 11.3 & 6.4 \\
\hline Nicaragua & 5.4 & 6.9 & 12.2 & 14.2 & 4.7 & 5.9 & 8.8 & 11.3 & 9.4 & 12.3 \\
\hline Niger & 0.33 & 0.89 & 1.1 & 3.4 & 2.5 & 5.3 & 1.8 & 4.1 & 5.3 & 11.5 \\
\hline Nigeria & 0.8 & 1.6 & 5.8 & 16.3 & 2.2 & 4.5 & 1.3 & 2.8 & 3.2 & 6.1 \\
\hline Norway & 46.3 & 22.3 & 43 & 16.1 & 33.8 & 15.3 & 7.7 & 3.6 & 8.1 & 4.2 \\
\hline Oman & 2.9 & 6.7 & 1.1 & 3.5 & 4.3 & 6.8 & 4.2 & 9.7 & 2.7 & 5.6 \\
\hline Pakistan & 7.4 & 11 & 3.3 & 5 & 2.7 & 3.7 & 2.2 & 3.1 & 2.5 & 3.6 \\
\hline Panama & 10.6 & 9.4 & 20 & 15.4 & 9.5 & 8.4 & 11.9 & 10.7 & 5.4 & 4.7 \\
\hline Papua New Guinea & 8.7 & 15.7 & 7.2 & 15.7 & 7.2 & 14 & 5.9 & 11.1 & 8.3 & 12.5 \\
\hline Paraguay & 16.4 & 18.5 & 13 & 13.9 & 8.1 & 9 & 4.4 & 4.9 & 3.3 & 3.7 \\
\hline Peru & 8.8 & 8.7 & 16.7 & 15.6 & 7.5 & 7.5 & 14.6 & 14.5 & 6.5 & 6.5 \\
\hline Philippines & 20.6 & 30.7 & 6.3 & 12.6 & 9 & 13.9 & 2.7 & 4.1 & 12.6 & 17.7 \\
\hline Poland & 93.1 & 49.5 & 31.3 & 14.5 & 44 & 22.6 & 20 & 10.7 & 7.1 & 3.9 \\
\hline Portugal & 75 & 33.5 & 38.6 & 10.6 & 51.3 & 18.7 & 28.4 & 11.4 & 20.1 & 8.9 \\
\hline Puerto Rico & 21.7 & 11.8 & 28.7 & 12.3 & 23.5 & 13.4 & 7.2 & 3.8 & 14.2 & 8.5 \\
\hline Qatar & 2.8 & 9.9 & 0.89 & 4.7 & 2.7 & 9 & 1.5 & 5.5 & 1.7 & 5.6 \\
\hline Republic of Moldova & 54.6 & 40.2 & 18.4 & 13.7 & 36.1 & 26.9 & 19.6 & 14.6 & 23.1 & 17.3 \\
\hline
\end{tabular}




\begin{tabular}{|c|c|c|c|c|c|c|c|c|c|c|}
\hline \multirow{3}{*}{ Site } & \multicolumn{10}{|c|}{ Age-standardized mortality rates } \\
\hline & \multicolumn{2}{|c|}{ Lung } & \multicolumn{2}{|c|}{ Prostate } & \multicolumn{2}{|c|}{ Colorectum } & \multicolumn{2}{|c|}{ Stomach } & \multicolumn{2}{|c|}{ Liver } \\
\hline & Crud Rate & ASR & Crud Rate & ASR & Crud Rate & ASR & Crud Rate & ASR & Crud Rate & ASR \\
\hline Romania & 82.6 & 45.7 & 26.1 & 10.8 & 39 & 19.2 & 20.9 & 10.6 & 21.1 & 11.3 \\
\hline Russian Federation & 66.6 & 44.3 & 21.4 & 13.6 & 29 & 18.9 & 25.6 & 17 & 9.6 & 6.4 \\
\hline Rwanda & 1.8 & 4.4 & 7.1 & 19 & 4.2 & 9.7 & 7.1 & 12.6 & 7.5 & 14.9 \\
\hline Saint Lucia & 17.1 & 13.6 & 55.7 & 34.5 & 11.4 & 8.2 & 15.9 & 11.6 & 3.4 & 2 \\
\hline Samoa & 21.6 & 28.6 & 13.7 & 16.6 & 7.8 & 10.9 & 9.8 & 13.3 & 4.9 & 7.1 \\
\hline Sao Tome and Principe & 9.6 & 18.2 & 6.7 & 15 & 1.9 & 6.6 & 7.7 & 11.7 & 6.7 & 14.8 \\
\hline Saudi Arabia & 3.1 & 5.1 & 0.77 & 1.7 & 5.6 & 7.3 & 1.5 & 2.3 & 3.3 & 5.8 \\
\hline Senegal & 1.5 & 3.6 & 7.9 & 21.6 & 2.1 & 4.9 & 3.1 & 6.8 & 8.5 & 18 \\
\hline Serbia & 112.7 & 61.1 & 29.6 & 13.1 & 45.9 & 23.3 & 15.1 & 7.9 & 12 & 6.3 \\
\hline Sierra Leone & 1.4 & 3.1 & 6.4 & 20 & 2.2 & 4.3 & 3.1 & 6.8 & 5.9 & 11.9 \\
\hline Singapore & 69.7 & 38.2 & 15.4 & 8.1 & 36.7 & 20.2 & 14.3 & 7.8 & 32.3 & 17.7 \\
\hline Slovakia & 66.3 & 38.2 & 36.6 & 19.7 & 51.9 & 29.5 & 14.3 & 8.3 & 11.4 & 6.7 \\
\hline Slovenia & 81.3 & 37.7 & 40.6 & 14.4 & 40.9 & 17.1 & 18.4 & 7.8 & 16.9 & 7.6 \\
\hline Solomon Islands & 7.3 & 14.1 & 3.8 & 6.9 & 3.5 & 6.7 & 1.3 & 2.3 & 13.6 & 20.7 \\
\hline Somalia & 1.6 & 3.6 & 3.6 & 9.7 & 3.6 & 7.9 & 2.1 & 4.8 & 1.6 & 3.5 \\
\hline South Africa & 18.6 & 27 & 15.6 & 27.9 & 6.7 & 10.2 & 3.3 & 4.8 & 5.3 & 7.2 \\
\hline South Sudan & 1.7 & 3.5 & 7.4 & 16.2 & 3.3 & 6.6 & 2.4 & 5 & 4 & 6.8 \\
\hline Spain & 77.2 & 34.4 & 25.5 & 7.4 & 44.1 & 16.8 & 15 & 6.1 & 17 & 7.6 \\
\hline Sri Lanka & 8.8 & 6.8 & 2.9 & 2.2 & 5.1 & 4 & 4.7 & 3.6 & 4 & 3.1 \\
\hline Sudan & 1.5 & 2.8 & 2.4 & 5.3 & 2.7 & 4.7 & 1.5 & 2.8 & 2.7 & 4.7 \\
\hline Suriname & 23.1 & 23.6 & 26.3 & 28.3 & 13.3 & 14.1 & 5.6 & 5.7 & 9.1 & 9.7 \\
\hline Swaziland & 1.6 & 4.4 & 7.9 & 22.9 & 1.6 & 3.5 & 0.3 & 1.1 & 2.8 & 5 \\
\hline Sweden & 36.9 & 15 & 50.4 & 15 & 32.7 & 12.4 & 6.7 & 2.7 & 10.4 & 4.5 \\
\hline Switzerland & 49 & 21.5 & 34.7 & 11.1 & 24.9 & 10.1 & 9.6 & 4.2 & 13.4 & 5.8 \\
\hline Syrian Arab Republic & 16.5 & 26.2 & 4.5 & 7.3 & 6.3 & 9.7 & 4.5 & 6.9 & 2.1 & 3.4 \\
\hline Tajikistan & 4.4 & 7.1 & 0.81 & 1.6 & 2.9 & 4 & 10.9 & 18.9 & 3.3 & 5.5 \\
\hline Tanzania, United Republic of & 0.37 & 0.84 & 9.3 & 22 & 2.4 & 5.5 & 1.7 & 3.8 & 3.9 & 8.2 \\
\hline Thailand & 41.5 & 27 & 8.6 & 5.3 & 14.7 & 9.7 & 5.2 & 3.4 & 48.3 & 32.3 \\
\hline The Netherlands & 71.7 & 29.7 & 33.5 & 11.7 & 40.5 & 16.5 & 9.8 & 4.1 & 7.6 & 3.3 \\
\hline $\begin{array}{l}\text { The former Yugoslav Republic of } \\
\text { Macedonia }\end{array}$ & 73 & 45.8 & 25.9 & 14.4 & 23.8 & 14.2 & 19.8 & 11.9 & 11.7 & 7.1 \\
\hline Timor-Leste & 7.3 & 14.5 & 1.5 & 3.5 & 3.4 & 7.1 & 2.4 & 5.2 & 4 & 7.4 \\
\hline Togo & 1.4 & 3 & 4.6 & 13.1 & 2.8 & 5.6 & 4.2 & 8.6 & 5.3 & 9.8 \\
\hline Trinidad and Tobago & 23.8 & 18.2 & 43.4 & 34.2 & 14.2 & 10.8 & 6.4 & 5 & 5 & 3.7 \\
\hline Tunisia & 28.8 & 25.5 & 7.3 & 5.7 & 8.6 & 7.5 & 6.2 & 5.4 & 2.6 & 2.3 \\
\hline Turkey & 70.7 & 68.6 & 12.8 & 11.9 & 13.8 & 13.1 & 15.8 & 15.1 & 6.8 & 6.5 \\
\hline Turkmenistan & 10.4 & 14.4 & 1.9 & 3.2 & 3 & 4.2 & 9.8 & 14.3 & 5.6 & 7.6 \\
\hline Uganda & 1.3 & 4.3 & 5.3 & 19.7 & 2.1 & 6.4 & 1.6 & 5.4 & 4.3 & 8.8 \\
\hline Ukraine & 61.9 & 38.5 & 24.4 & 13.8 & 34 & 20.3 & 24.9 & 15.3 & 6.2 & 3.9 \\
\hline
\end{tabular}




\begin{tabular}{|c|c|c|c|c|c|c|c|c|c|c|}
\hline \multirow{3}{*}{ Site } & \multicolumn{10}{|c|}{ Age-standardized mortality rates } \\
\hline & \multicolumn{2}{|c|}{ Lung } & \multicolumn{2}{|c|}{ Prostate } & \multicolumn{2}{|c|}{ Colorectum } & \multicolumn{2}{|c|}{ Stomach } & \multicolumn{2}{|c|}{ Liver } \\
\hline & Crud Rate & ASR & Crud Rate & ASR & Crud Rate & ASR & Crud Rate & ASR & Crud Rate & ASR \\
\hline United Arab Emirates & 2.1 & 6.3 & 0.73 & 4.4 & 3 & 7.7 & 0.97 & 3.8 & 0.89 & 4.2 \\
\hline United Kingdom & 60.6 & 25.2 & 40 & 12.7 & 34 & 13.3 & 8.7 & 3.5 & 12.3 & 5.3 \\
\hline United States of America & 50.3 & 25.9 & 17.7 & 7.7 & 17.7 & 9.6 & 4.3 & 2.3 & 12.7 & 7.2 \\
\hline Uruguay & 63.6 & 42 & 34.5 & 17.1 & 33.1 & 19.3 & 16.9 & 10.6 & 4.8 & 3.1 \\
\hline Uzbekistan & 8.7 & 11.6 & 2.1 & 3.1 & 3.9 & 5.1 & 7.9 & 10.8 & 4.1 & 5.5 \\
\hline Vanuatu & 9.1 & 15.6 & 6.3 & 10.4 & 2.8 & 4.3 & 1.4 & 2.2 & 15.4 & 20.2 \\
\hline Venezuela, Bolivarian Republic of & 16.9 & 18.2 & 21.1 & 23.3 & 7.5 & 8.1 & 7.2 & 7.7 & 3.9 & 4.2 \\
\hline Viet Nam & 30.9 & 31.6 & 3.9 & 3.7 & 8.6 & 8.7 & 19.7 & 19.7 & 41.3 & 39.1 \\
\hline Yemen & 1.8 & 3.9 & 0.27 & 0.68 & 3.3 & 7.5 & 3.7 & 8.5 & 2.7 & 6.2 \\
\hline Zambia & 1.3 & 4 & 9.1 & 28.4 & 1.2 & 3.2 & 1.2 & 3.4 & 1.2 & 2.7 \\
\hline Zimbabwe & 2.1 & 5.8 & 11.4 & 29.7 & 3.3 & 8.4 & 2.5 & 6.8 & 3.9 & 6.8 \\
\hline
\end{tabular}

Prostate

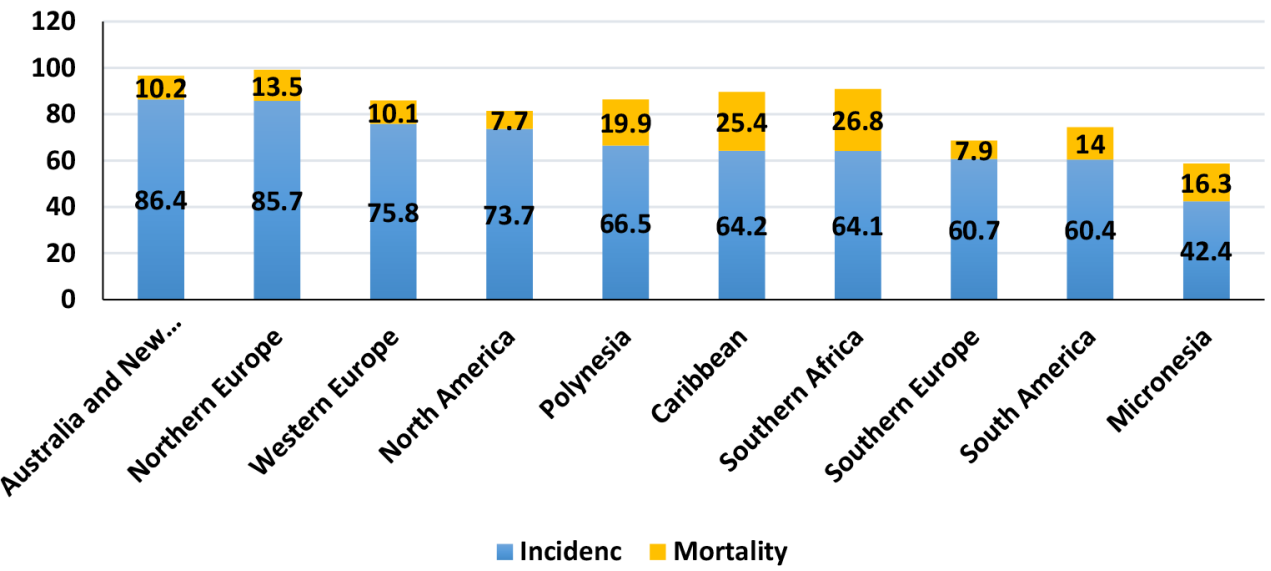

Fig. 8 Bar chart of region-specific incidence and mortality age-standardized rates for prostate cancer in males 2018. Rates are shown in descending order of the world $(W)$ age-standardized rate.

Source: GLOBOCAN 2018.

\section{Colorectum Cancer}

CRC is the third-most diagnosed cancer and is the fourth leading cause of death worldwide, with around 1.4 million new cases and nearly 700,000 deaths recorded in 2012 due to CRC. ${ }^{20}$ In most countries, the incidence of CRC has increased. ${ }^{21}$ According to demographic forecasts, the global CRC is expected to increase by $60 \%$, bringing more than 2.2 million new cases and 1.1 million deaths from this cancer by $2030{ }^{22}$ The disease can be seen as a sign of economic development. In countries undergoing major development shifts, cancer incidences boost with increases in the HDI. ${ }^{21}$ An increase in incidence is associated with generations, changes in diet patterns, obesity, and lifestyle factors. Mortality rates is seen more in developed countries, reflecting improvements in survival through the adoption of best practices for treating and managing cancer in the developed countries.

According to the results of the cancer record in 2018, 184,518 cases (about $10.2 \%$ of all cancers) were recorded for
CRC in both genders. The highest incidence of CRC based on world areas in men was in Australia and New Zealand (41.7 per 100,000), Southern Europe (40.4 per 100,000), and Central and Eastern Europe (37.5 per 100,000), respectively. The highest incidence is reported from the Central and Eastern Europe (20.5 per 100,000), Southern Europe (15.4 per 100,000), and Northern Europe (13.5 per 100,000) (Fig. 10). According to cancer record results in 2018, Hungary (70.6 per 100,000), Slovakia (60.7 per 100,000), and Republic of Korea, (59.5 per 100,000) have the highest incidence of CRC (Table 1, Fig. 11).

According to the results of 2018, the highest mortality rates for CRC were in males from Hungary $(31.2 \%$ in 100,000), Slovakia $(29.5 \%$ in 100,000$)$, and Croatia $(27.4 \%$ in 100,000$)$ (Table 2).

\section{Stomach Cancer}

Gastric cancer is recognized as the fourth most common cancer in the world, and is the second leading cause of cancer 


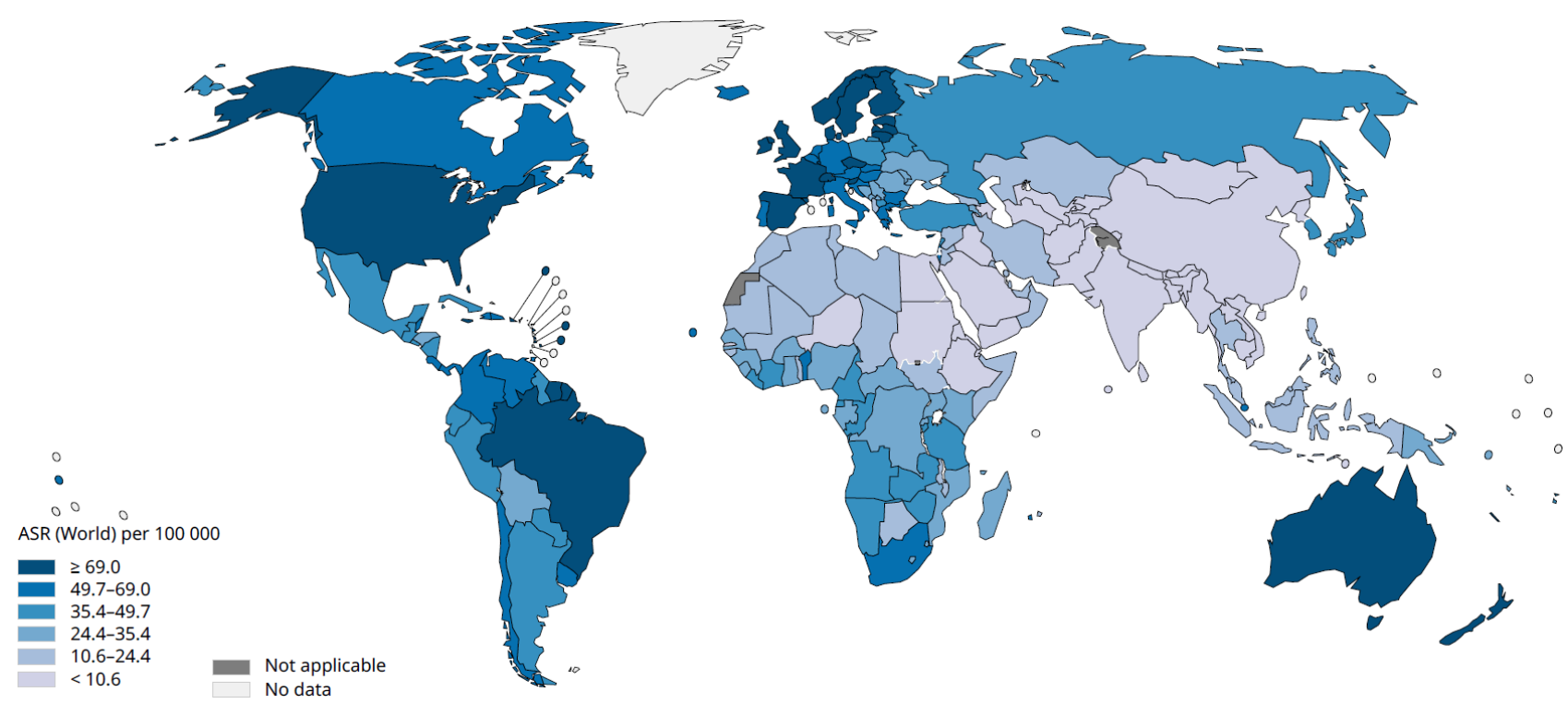

Fig. 9 Global map presenting age-standardized incidence rates by world countries for prostate cancer in males in 2018. Source: GLOBOCAN 2018.

\section{Colorectum}

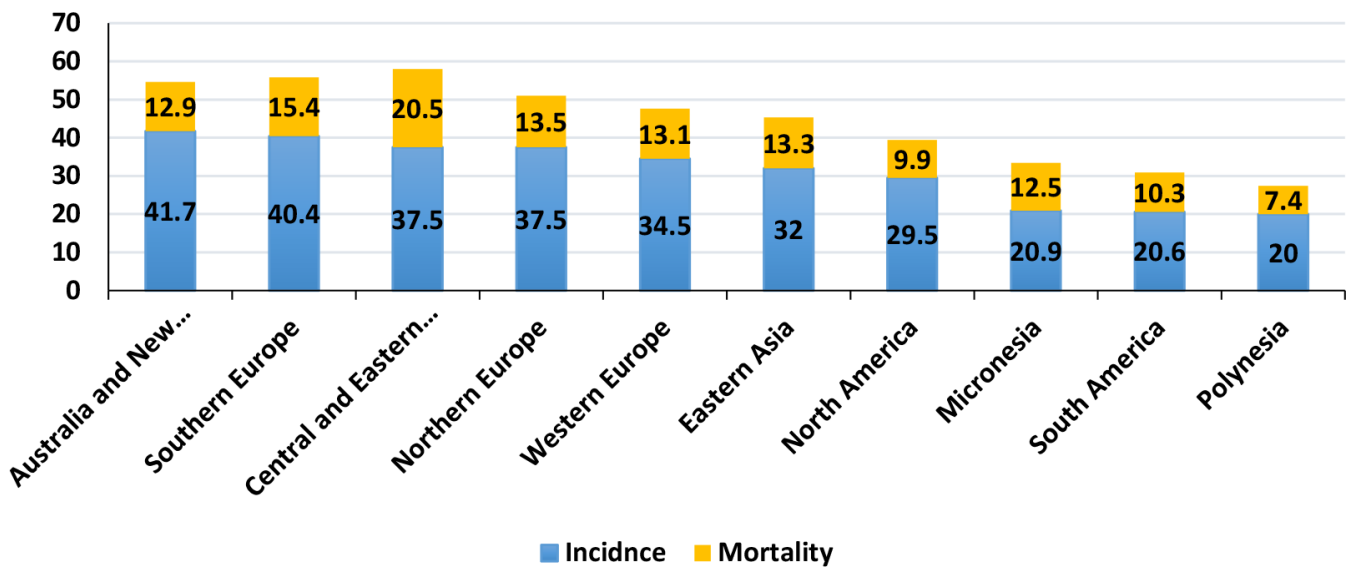

Fig. 10 Bar chart of region-specific incidence and mortality age-standardized rates for colorectum cancer in males 2018. Rates are shown in descending order of the world $(W)$ age-standardized rate.

Source: GLOBOCAN 2018.

death. The incidence of this cancer is due to the process of creating a cancerous tumor in stomach in several stages and, therefore, it is classified as a multifactorial disease. The reason for the development of the cancer is the presence of infectious, environmental, and zoonotic agents in humans. ${ }^{23-25}$ The prevalence of gastric cancer varies among populations. Genetic and lifestyle variations, especially in dietary habits - such as salt intake - and the difference in detection time, have led to varying cancer incidence in parts of the world which seem to be due to the environmental and racial differences. ${ }^{26}$ Gastric cancer is common in countries like China, Chile, Ireland, Costa Rica, northern and southern Korea, Finland, and Iceland. ${ }^{27}$

According to a cancer record in 2018, gastric cancer was identified as the fifth most common cancer in 2018 with $1,033,701$ cases (5.7\% of total cancers), of which 683,754 cases were in men. Gastric cancer, with 78,285 deaths after lung cancer, was the second cause of death due to cancer in both genders, of which 513,555 cases were in males. According to the world areas, the highest incidence of gastric cancer in men was
Eastern Asia (1.32 per 100,000), Central and Eastern Europe (17.1 per 100,000), and South America (12.7 per 100,000), and the highest mortality rate was reported for Eastern Asia (23 per 100,000), Central and Eastern Europe (14 per 100,000), and Western Asia (10 per 100,000) (Fig. 12). The results showed that the highest incidence was related to the countries of the Republic of Korea (57.8 per 100,000), Mongolia (47.2 per 100,000), and Japan $(40.7$ per 100,000) (Table 1, Fig. 13). According to the results of 2018, the highest mortality rates for gastric cancer were in males from Mongolia (36.7 per 100,000), Kyrgyzstan (6.26 per 100,000), and China (25 per 100,000) (Table 2).

\section{Cancer Liver}

Primary liver cancer is the fifth most common cancer and the third cause of death from cancer in the world. In most countries, primary carcinoma of the liver cells accounts for about $90-75 \%$ of liver cancers. It affects both sexes, but it is more prevalent in men (16 per 100,000) than in women (6 per 100,000). The incidence of liver cancer is elevated with age, 


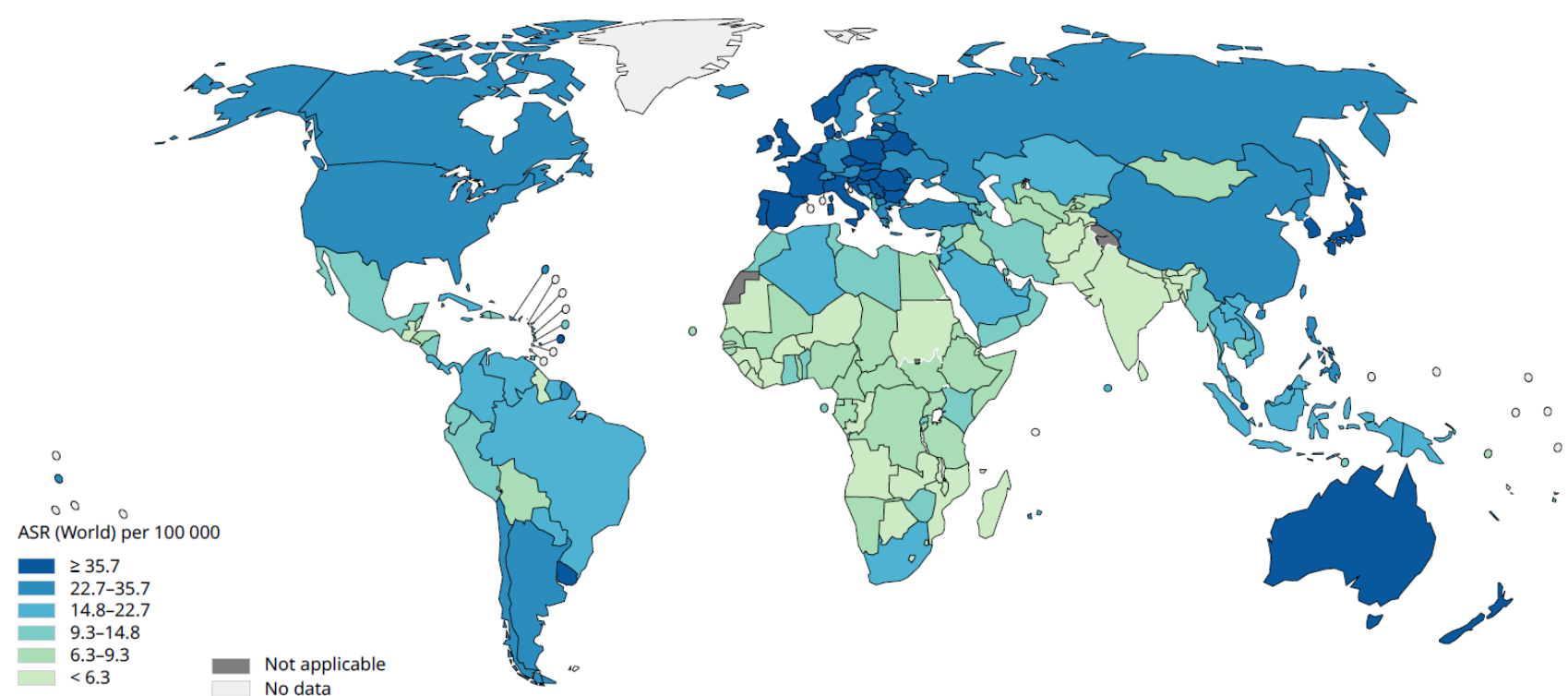

Fig. 11 Global map presenting age-standardized incidence rates by world countries for colorectum cancer in males in 2018. Source: GLOBOCAN 2018.

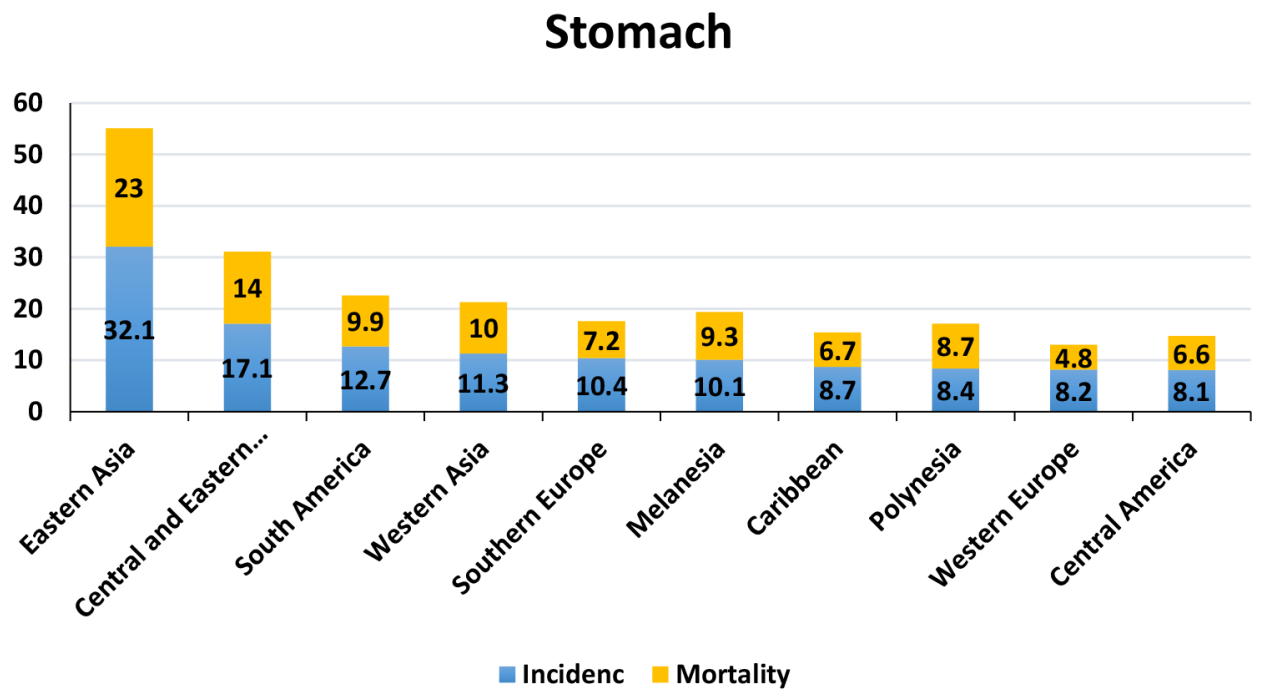

Fig. 12 Bar chart of region-specific incidence and mortality age-standardized rates for stomach cancer in males 2018. Rates are shown in descending order of the world $(\mathrm{W})$ age-standardized rate.

Source: GLOBOCAN 2018.

the highest incidence prevails among the age group of 70-60 years. The rate of liver cancer has been reported from 10 cases per 100,000 people in North America and Western Europe to 150 cases per 100,000 people in Africa and $50-70$ cases in Asia. Therefore, the highest incidence of liver cancer are reported from the regions of East Asia, Southeast, and Central and Eastern Africa and the lowest incidence is in advanced countries (except South America). ${ }^{28-30}$ The most important causes of liver cancer, in more than $75 \%$ of cases, are chronic hepatitis $\mathrm{C}$ and $\mathrm{B}$ infections, which can be prevented. ${ }^{31}$

Based on cancer record results in 2018, a total of 841,080 cases $(7.4 \%$ of all cancers) were related to liver cancer, of which 596,574 cases were in men. According to the same results, 781,631 of deaths are due to liver cancer of which 548,375 were reported for men. According to world areas, the highest incidence of liver cancer in men was related to Easter Asia (26.8 per 100,000$)$, Micronesia (25.6 per 100,000$)$, and South-Eastern Asia (21 per 100,000), and the highest rates of mortality associated with Easter Asia (2.24 in 100,000), South-Eastern Asia (20.20 in 100,000) and North Africa (4.20 in 100,000) (Fig. 14). The results also showed that the highest incidence of liver cancer is in males from Mongolia (98.4\% in 100,000$)$, Egypt $(48.4 \%$ in 100,000$)$, and Vietnam $(39.1$ in 100,000) (Table 1, Fig. 15).

According to the results of 2018, the highest mortality rate of liver cancer in men was in Mongolia (98.4\% in 100,000), Egypt $(48.4 \%$ in 100,000$)$, and The Gambia $(41.1$ in 100,000$)$ (Table 2).

The results of our study revealed that there was a positive and significant correlation between the incidence of lung cancer $(\mathrm{R}=0.629, P<0.0001)$, prostate $(\mathrm{R}=0.534, P<0.0001)$, colorectal $(\mathrm{R}=0.745, P<0.0001)$, and stomach $(\mathrm{R}=0.268, P<$ 0.001 ) with HDI index, while there was no significant relationship between liver cancer and HDI index $(\mathrm{R}=0.079, P>0.05)$.

The results also showed that there was a positive and significant correlation between the mortality from lung cancer 


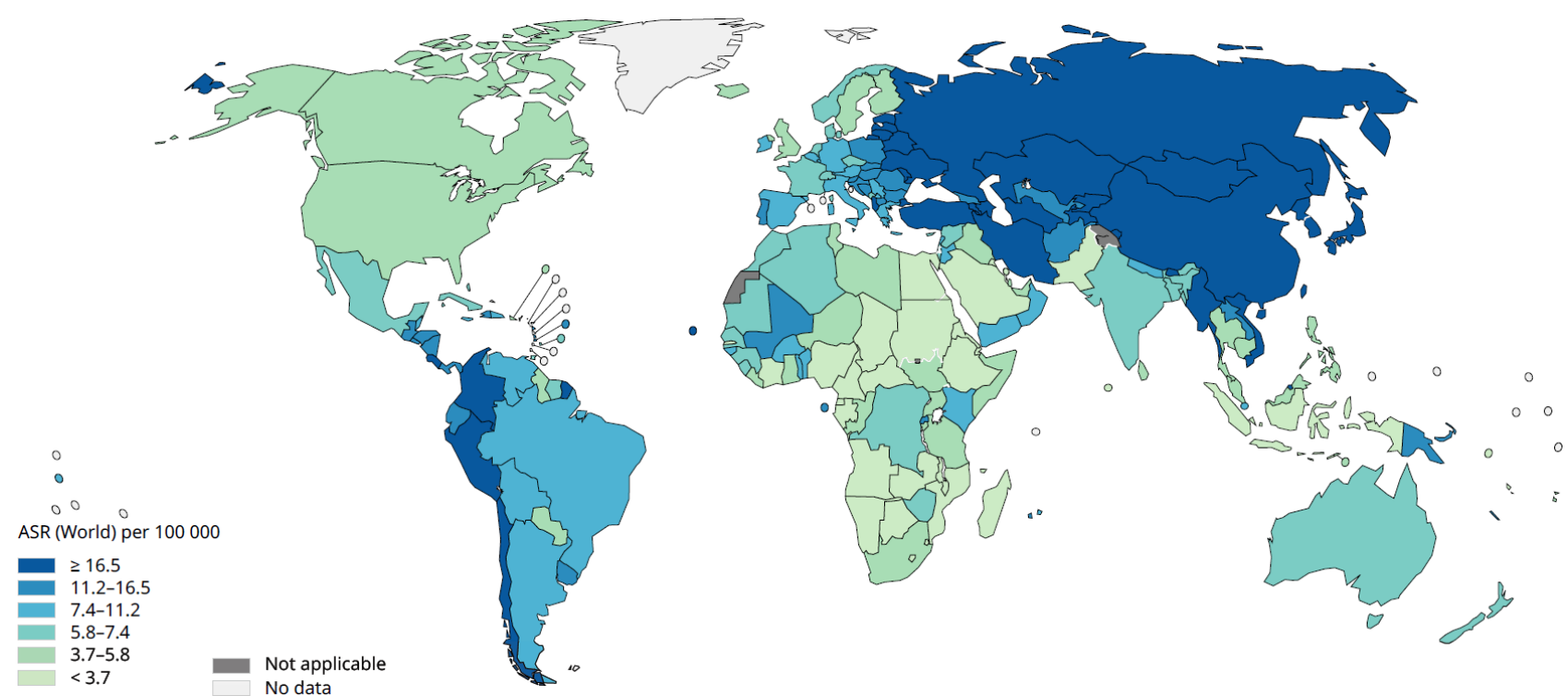

Fig. 13 Global map presenting age-standardized incidence rates by world countries for stomach cancer in males in 2018. Source: GLOBOCAN 2018.

\section{Liver}

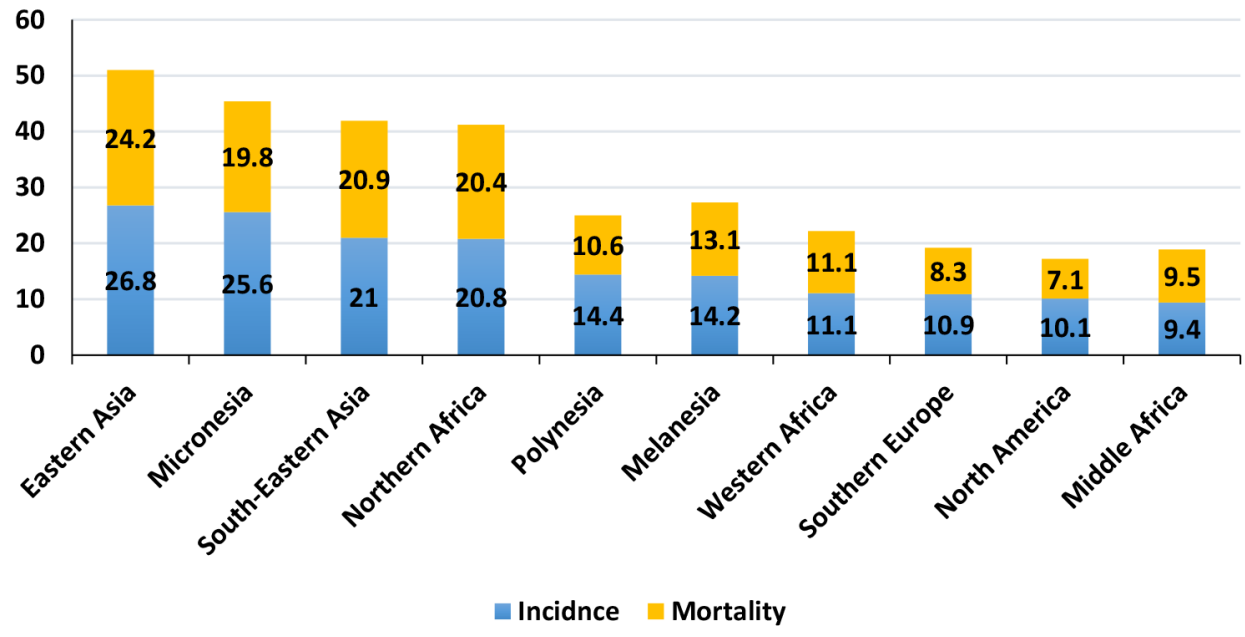

Fig. 14 Bar chart of region-specific incidence and mortality age-standardized rates for liver cancer in males 2018. Rates are shown in descending order of the world $(W)$ age-standardized rate.

Source: GLOBOCAN 2018.

$(\mathrm{R}=0.632, P<0.0001)$ and colorectal $(\mathrm{R}=0.627, P<0.0001)$ with HDI, whereas this correlation in prostate cancer was negative $(\mathrm{R}=-0.187, P<0.01)$ and there was no significant correlation between mortality and the HDI in liver and stomach cancers $(P>0.05)$.

\section{Summary and Conclusions}

The main reasons for increasing cancer cases in developing countries include population growth, an increase in the percentage of elderly people that is associated with an increase in annual cancer incidence and an increase in the incidence and cancer burden in the future. ${ }^{32}$

It is estimated that the number of new cases of cancer will be approximately 26.4 million and the number of deaths from cancer will reach about 17 million by 2030. Population aging, improving quality of life, technological advancements, lifestyle changes, and cancer risk factors are among the factors that cause cancer deaths to reach 62,000 by $2020 .^{32,33}$

Studies have shown that HDI can be used as a predictor of lung cancer. High life expectancy and higher mean longevity are the main reasons for increased LC in regions with very high HDI. Since aging cannot be controlled, the risk of developing lung cancer cannot be prevented as well. Other notable points in areas with high HDI are the increased prevalence of smoking in both genders, especially in young people. In highHDI societies, the problem of air pollution and exposure to pollutants such as aromatic cyclic hydrocarbons, which are an environment friendly carcinogen, is associated with increased lung cancer, especially in the elderly. In previous studies, a positive and direct correlation between lung cancer and the annual average of dust has been confirmed. Therefore, lung cancer with the highest global incidence and mortality and its upward trend by 2040 worldwide is significant. The human 


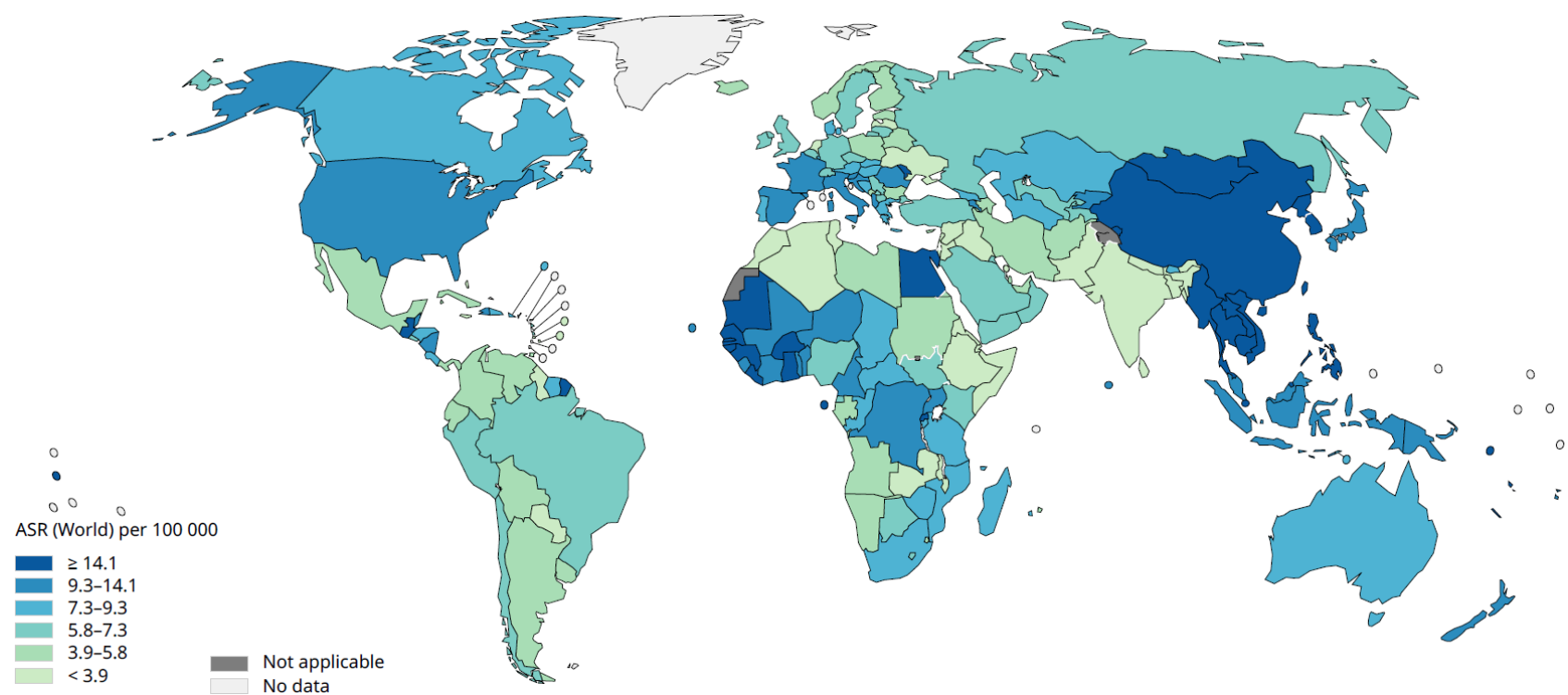

Fig. 15 Global map presenting age-standardized incidence rates by world countries for liver cancer in males in 2018.

\section{A) Lung cancer}

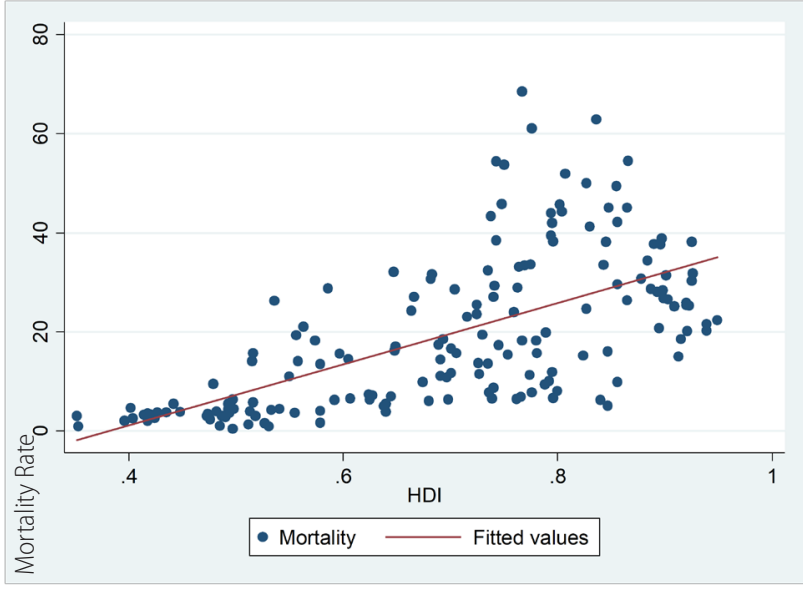

R Sq. linear $=0.629, P<0.0001$

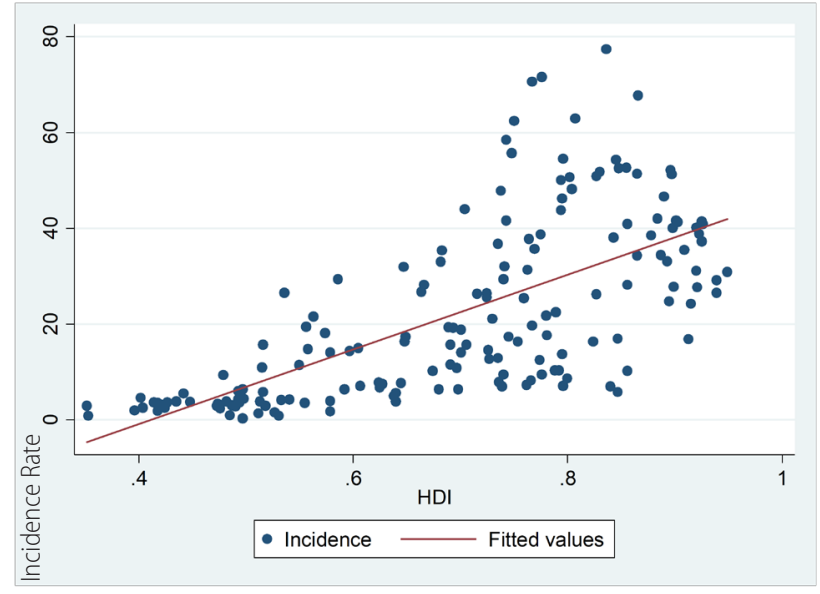

R Sq. linear $=0.632, P<0.0001$

B) Prostate cancer

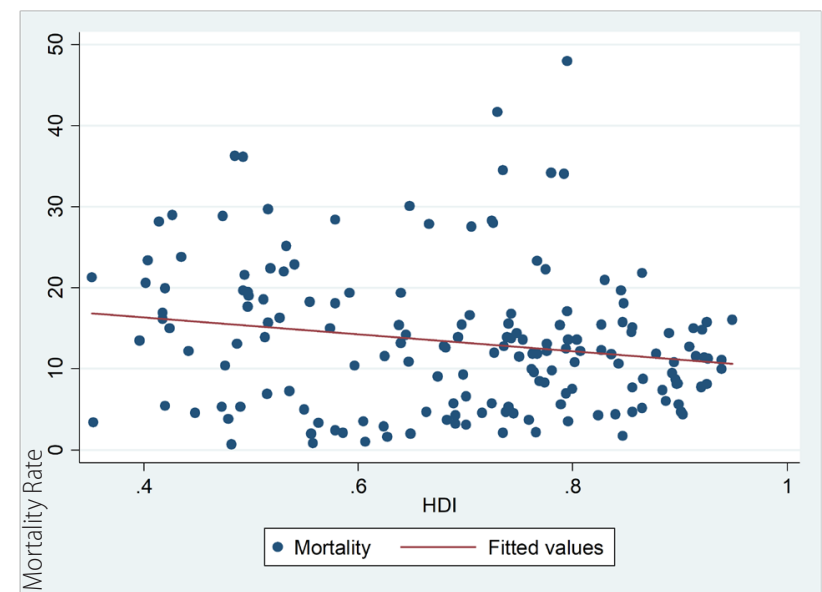

R Sq. linear $=0.534, P<0.0001$

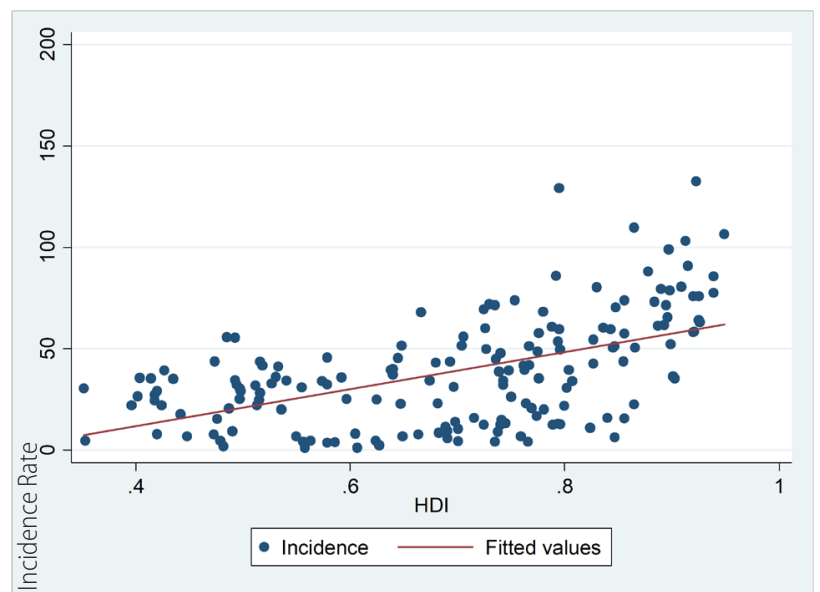

R Sq. linear $=-0.187, P<0.01$

Fig. 16 Correlation between incidence and mortality rates with human development index for the five most common cancers in males in the world in 2018. 


\section{C) Colorectum cancer}

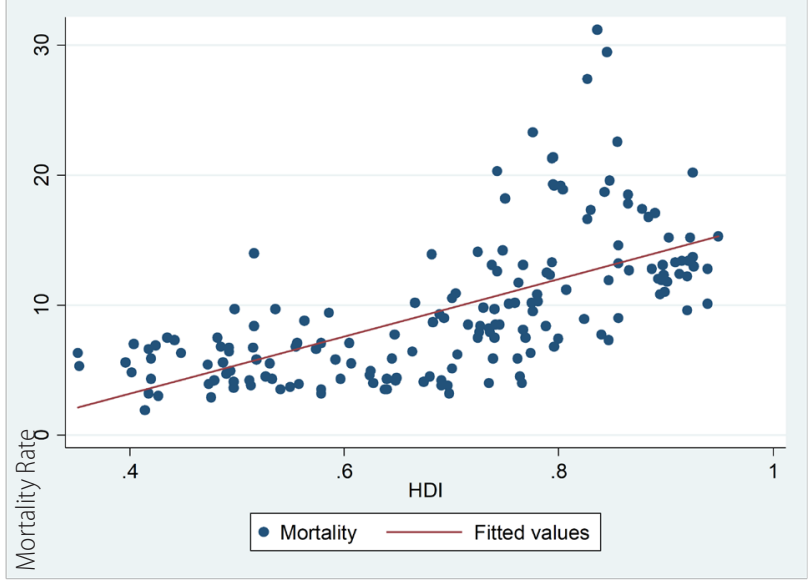

R Sq. linear $=0.745, P<0.0001$

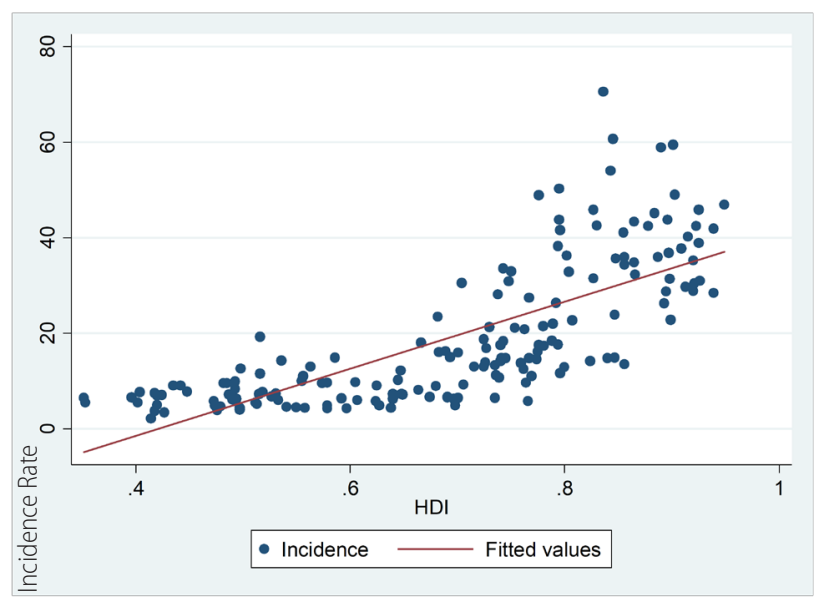

RSq. linear $=0.627, P<0.0001$

\section{D) Stomach cancer}

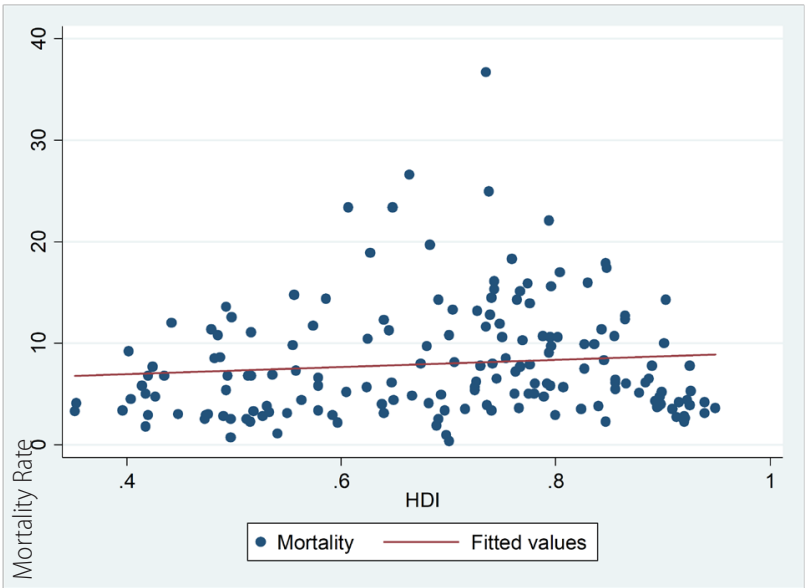

R Sq. linear $=0.268, P<0.0001$

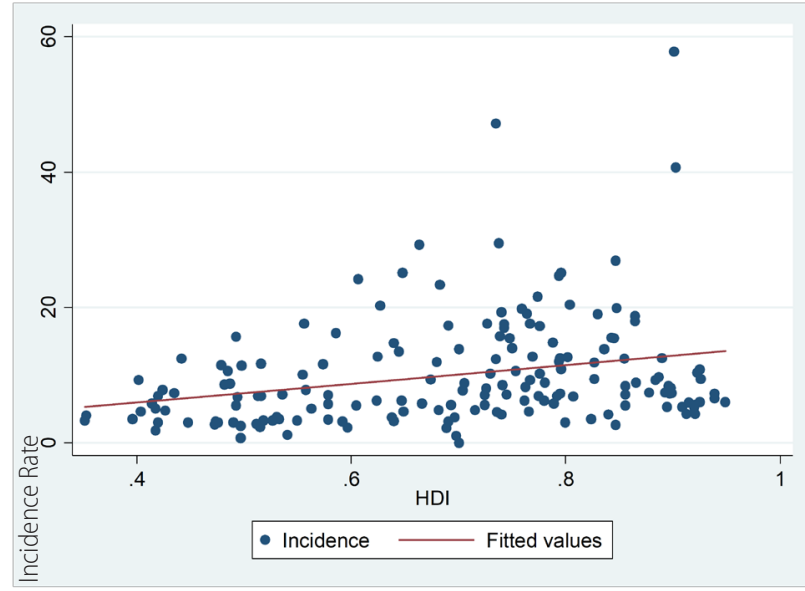

R Sq. linear $=0.098, P>0.05$

E) Liver cancer

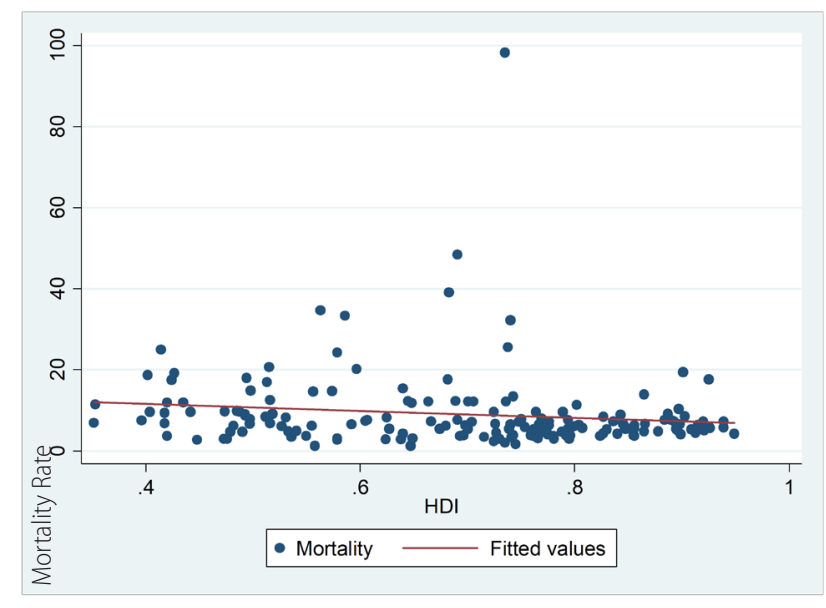

R Sq. linear $=-0.079, P>0.05$

\section{Fig. 16 Continued.}

development index, as an important factor, can be effective in reducing the incidence and mortality of the patient. Reducing smoking and tobacco and not being exposed to other risk factors such as asbestos, radon, and air pollution are reasonable decisions in this respect. ${ }^{6,34,35}$

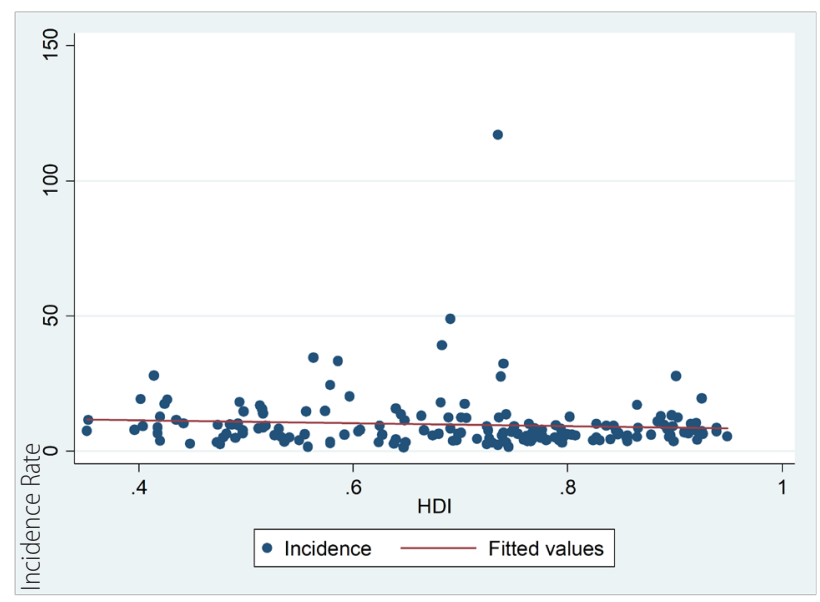

R Sq. linear $=-0.138, P>0.05$

Prostate cancer often affects the elderly and continues to be highly fatal. In most cases, prostate cancer is formed not only because of biological and inherent characteristics of an individual, but also because of environment where they live in. Significant and rapid changes in the incidence of prostate 
cancer, long duration, and its potential require a more serious attention to the environmental and lifestyle factors affecting individuals. The difference in the rate of prostate cancer among men in developed countries and in Asian countries is a feedback on their different lifestyles. Other causes of difference in these two categories of people are dietary, sexual, and behavior patterns, alcohol consumption and exposure to ultraviolet ${ }^{36}$ radiation. Recent studies have shown that in countries with high and very high HDI, new prostate cancer cases are on the rise, while mortality rates in these countries are lower than in poor countries or those with lower HDIs (such as African and Caribbean countries). One of the main reasons for this difference is the easier access to screening and modern treatments in countries with high and very high HDI. ${ }^{37,38}$

One of the reasons for the high incidence of CRC in countries with a high HDI can be the presence of risk factors for CRC in these countries. ${ }^{16,39,40}$ The low mortality rate of CRC in countries with a low HDI can be due to low levels of CRC as the result of lack of proper diagnosis and failure to record and report this cancer. ${ }^{41-43}$ Another reason for the difference in the incidence and mortality of CRC with the country's HDI is the existence and use of screening and diagnostic tests, such as sigmoidoscopy and colonoscopy. ${ }^{44,45}$ Certainly, changes in lifestyle and westernization factors are likely to be partly responsible for global changes in the incidence of CRC, as well as the increasing trend of incidence in developing countries. These significant risk factors include alcohol intake, ${ }^{46,47}$ inappropriate diet (low intake of fruits and vegetables and high consumption of red meat/processed meat), ${ }^{48,49}$ obesity, ${ }^{50,51}$ physical inactivity, ${ }^{52}$ and cigarettes, ${ }^{53-55}$ with which they can control the incidence and mortality of cancers, including CRC.

Prevention and treatment of gastric cancer, which is currently one of the most common malignancies worldwide, continues to be a problem. ${ }^{38}$ The rate of gastric cancer in men is twice as high as women and varies from country to country. ${ }^{56}$ Regional variations in the incidence of this cancer indicate differences in food patterns, consumed food and availability of fresh produce, and the prevalence of Helicobacter pylori infection. Repeated infections with $H$. pylori are one of the most important risk factors for detecting gastric cancer, and $90 \%$ of new cases of gastric cancer worldwide are associated with this bacterium. ${ }^{57}$ Proximal tumors are more common in developed countries and higher social classes. Therefore, the incidence is higher. One of the reasons for a higher incidence in higher HDI countries may be the difference in diagnostic programs in high-HDI countries compared to low-HDI ${ }^{58}$ countries. Some foods also have a natural nitrate concentration (cabbage, cauliflower, carrots, celery, radishes, beets, and spinach). Nitrate content in fertilizer, water, and soil also increases nitrate in the diet that can be effective in gastric cancer. ${ }^{59}$ Therefore, lifestyle modification is a practical strategy for preventing gastric cancer, especially among the elderly. ${ }^{59,60}$

Hepatocyte carcinoma (HCC) is of global importance due to its high rate of progression and high mortality rates. Almost $85 \%$ of LC cases and deaths occur in developing countries and significantly in countries with low and moderate HDIs. It can be said that these countries are often in the process of industrialization, and this affects all aspects of their lives, including those related to health. In countries with low and intermediate HDI, HCC risk factors, including diabetes mellitus, ${ }^{36}$ metabolic syndrome, obesity, ${ }^{38}$ alcoholic beverages, ${ }^{39}$ and HBV and HCV infection are more prevalent, leading to an increased incidence of hepatocellular carcinoma. The incidence and mortality rate of liver cancer in countries with high prevalence, like Japan and China, is declining, which can be attributed to lower levels of aflatoxin contamination, immunization against $\mathrm{HBV}$, and other cancer prevention programs. Reducing chronic schistosomiasis, more health measures in the transmission and donation of blood to prevent $\mathrm{HCV}$ infection, and the implementation of policies to prevent unwanted intravenous infusion has also been effective.

\section{Conflict of Interest}

None.

\section{Financial support}

Yazd University of Medical Sciences, Yazd, Iran.

\section{Acknowledgements}

We thank cancer registries worldwide for their collaboration [GLOBOCAN], without their efforts, there would be no global cancer estimates.

\section{References}

1. Ciobanu LG, Ferrari AJ, Erskine HE, Santomauro DF, Charlson FJ, Leung J, et al. The prevalence and burden of mental and substance use disorders in Australia: Findings from the Global Burden of Disease Study 2015. Austr. New Zeal J Psychiatry. 2018;12(11):e317.

2. Mohebbi E, Nahvijou A, Hadji M, Rashidian H, Seyyedsalehi MS, Nemati S, et al. Iran Cancer Statistics in 2012 and projection of cancer incidence by 2035. Basic Clin Cancer Res. 2018;9(3):e235.

3. Goodarzi E Khazaei Z, Moayed L, Adineh H, Sohrabivafa M, Darvishi 1, Dehghani S. Epidemiology and population attributable fraction of melanoma to ultraviolet radiation in Asia: an ecological study. WCRJ. 2018;5(2):e1114.

4. Zargani A, Nasiri M, Hekmat K, Abbaspour Z, Vahabi S. A Survey on the relationship between religiosity and quality of life in patients with breast cancer: A study in Iranian Muslims. Asia-Pac J Oncol Nurs. 2018;5(2):e217.

5. Vaisy A, Lotfinejad S, Zhian F. Relationship between utrine cervical carcinoma and oral contraceptives. J Gorgan Univ Med Sci. 2012;14(3):e258

6. Öberg M, Jaakkola MS, Woodward A, Peruga A, Prüss-Ustün A. Worldwide burden of disease from exposure to second-hand smoke: A retrospective analysis of data from 192 countries. The Lancet. 2011;377(9760):139-46.

7. Stewart B, Wild CP. World cancer report 2014. Health. 2017; 15(3):e856.

8. Khazaei S, Mansori K, Soheylizad M, Gholamaliee B, Shadmani FK, Khazaei Z, et al. Epidemiology of lung cancer in Iran: Sex difference and geographical distribution. Middle East J Cancer. 2017;8(4):223-8.

9. Bagnardi V, Blangiardo M, La Vecchia C, Corrao G. A meta-analysis of alcohol drinking and cancer risk. Br J Cancer. 2001;85(11):e1700

10. Mirzaei M, Sharifnia G, Khazaei Z, Sadeghi E, Fallahzadeh H, Namayandeh SM. Prevalence of general obesity and central adiposity and its related factors in adult population of Yazd. SSU_J. 2017;25(9):736-47.

11. Nassehinia H, Kiani M, Khazaei Z, Torabi H, Sohrabivafa M, Beiranvand R, et al. Comparing efficiency of rice washing and soaking processes in reducing the amount of aflatoxin B1. Iran J Health Safety Environ. 2017:5(1):938-4.

12. Norouzirad R, Khazaei Z, Mousavi M, Adineh HA, Hoghooghi M, Khabazkhoob M, et al. Epidemiology of common cancers in Dezful county, southwest of Iran. Immunopathol Persa. 2017;4(1):e369

13. Bray F, Ferlay J, Soerjomataram I, Siegel RL, Torre LA, Jemal A. Global cancer statistics 2018: GLOBOCAN estimates of incidence and mortality worldwide for 36 cancers in 185 countries. CA Cancer J Clin 2018;6:394-424

14. GLOBOCAN 2018. Available from: http://www.gco.iarc.fr/today/datasourcesmethods. [Last accessed on 2018 Jan 17]. 
15. Goodarzi E, Moslem A, Feizhadad H, Jarrahi AM, Adineh HA, Sohrabivafa $M$, et al. Epidemiology, incidence and mortality of thyroid cancer and their relationship with the human development index in the world: An ecology study in 2018. Adv Human Biol. 2019;9(2):e162

16. Bray F, Jemal A, Grey N, Ferlay J, Forman D. Global cancer transitions according to the Human Development Index (2008-2030): a populationbased study. Lancet Oncol. 2012;13(8):790-801.

17. Khazaei S, Rezaeian S, Khazaei Z, Molaeipoor L, Nematollahi S, Lak P, et al. National breast cancer mortality and incidence rates according to the human development index: an ecological study. Adv Breast Cancer Res. 2016;5(01):e30.

18. Programme UND. Human Development Report 2016. http://hdr.undp.org/ en [accessed January 2018].

19. Khazaei Z, Ghorat F, Jarrahi A, Adineh H, Sohrabivafa M, Goodarzi E. Global incidence and mortality of skin cancer by histological subtype and its relationship with the human development index(HDI): An ecology study in 2018. World Cancer Res J. 2019;6(2):e13.

20. Ferlay J, Soerjomataram I, Ervik M, Dikshit R, Eser S, Mathers C, et al. GLOBOCAN 2012 v1. 0, Cancer Incidence and Mortality Worldwide: IARC CancerBase No. 11. Lyon, France: International Agency for Research on Cancer. 2013; 12(7);e2563.

21. Fidler MM, Soerjomataram I, Bray F. A global view on cancer incidence and national levels of the human development index. Int J Cancer. 2016;139(11):2436-46.

22. Arnold M, Sierra MS, Laversanne M, Soerjomataram I, Jemal A, Bray F. Global patterns and trends in colorectal cancer incidence and mortality. Gut. 2017;66(4):683-91.

23. Zabaleta J. Multifactorial etiology of gastric cancer. Cancer Epigenetics: Springer; 2012. p. 411-35.

24. Jemal A, Bray F, Center MM, Ferlay J, Ward E, Forman D. Global cancer statistics. CA Cancer J Clin. 2011;61(2):69-90.

25. Khazaei Z, Jarrahi AM, Momenabadi V, Ghorat F, Adineh H, Sohrabivafa M, et al. Global cancer statistics 2018: global estimate of incidence and mortality worldwide stomach cancer and their relationship with the human development index (HDI). World Cancer Res J. 2019;6:9.

26. IRAVANI S. Gastric cancer as a multifactorial disease. 2013; 12(9):e256.

27. Brenner H, Rothenbacher D, Arndt V. Epidemiology of Stomach Cancer. Cancer Epidemiology: Springer; 2009. p. 467-77.

28. Ferlay J, Soerjomataram I, Dikshit R, Eser S, Mathers C, Rebelo M, et al. Cancer incidence and mortality worldwide: Sources, methods and major patterns in GLOBOCAN 2012. Int J Cancer. 2015;136(5):359-86.

29. Wong MC, Jiang JY, Goggins WB, Liang M, Fang Y, Fung FD, et al. International incidence and mortality trends of liver cancer: A global profile. Scient Rep. 2017;7(3):e45846.

30. Goodarzi E, Ghorat F, Jarrahi AM, Adineh H, Sohrabivafa M, Khazaei Z. Global incidence and mortality of liver cancer and its relationship with the human development index (HDI): An ecology study in 2018. World Cancer Res J. 2019;6(2);e1255

31. Moradi G, Goodarzi E, Khazaei Z. Prevalence of Hepatitis B and C in prisons worldwide: A meta-analysis during the years 2005-2015. Biomed. Res. Ther. 2018;5(4):2235-51.

32. Karami K, Cheraghi M, Amori N, Pedram M, Sobhani A. Common cancers in Khuzestan province, south west of Iran, during 2005-2011. Asian Pac J Cancer Prev: APJCP. 2014;15(21):9475-8

33. Amori N, Aghajani M, Asgarian F, Jazayeri M. Epidemiology and trend of common cancers in Iran (2004-2008). Eur J Cancer Care. 2017;26(5):e318.

34. Soheylizad M, Khazaei S, Khazaei S, Rezaeian S. Relation between lung cancer incidence and mortality rates with human development index and its components: A global ecological study. Iran J Cancer Prev 2016;9(5):e256

35. IWGotEoCRt H. Organization WH, Cancer IAfRo: Smokeless Tobacco and Some Tobacco-Specific N-Nitrosamines, vol. 89. Lyon, France: World Health Organization. 2007;11(6):e896.

36. Sadeghi-Gandomani H, Yousefi M, Rahimi S, Yousefi S, Karimi-Rozveh A, Hosseini S, et al. The incidence, risk factors, and knowledge about the prostate cancer through worldwide and Iran. World Cancer Res J. 2017;4(4):1-8.

37. Priyadarshi V. Clinical epidemiology of carcinoma of prostate: An institutional experience. Urol Nephrol Open Access J Clin Epidemiol Carcin Prost Instit Exp. 2016:;23(6):e158
38. Ferlay J, Shin HR, Bray F, Forman D, Mathers C, Parkin DM. Estimates of worldwide burden of cancer in 2008: GLOBOCAN 2008. Int J Cancer. 2010;127(12):2893-917.

39. Ferrari F, Reis MAM. Study of risk factors for gastric cancer by populational databases analysis. World J Gastroenterol WJG. 2013;19(48):e9383.

40. Liu Y, Arai A, Obayashi Y, Kanda K, Boostrom E, Lee RB, et al. Trends of gender gaps in life expectancy in J apan, 1947-2010: Associations with gender mortality ratio and a social development index. Geriat Gerontol Int. 2013;13(3):792-7.

41. Coleman MP, Quaresma M, Berrino F, Lutz J-M, De Angelis R, Capocaccia $R$, et al. Cancer survival in five continents: a worldwide population-based study (CONCORD). Lancet Oncol. 2008;9(8):730-56.

42. Jung K-W, Won Y-J, Kong H-J, Oh C-M, Shin A, Lee J-S. Survival of Korean adult cancer patients by stage at diagnosis, 2006-2010: National cancer registry study. Cancer Res Treat Off J Kor Cancer Assoc. 2013;45(3):e162.

43. Mallin K, Palis BE, Watroba N, Stewart AK, Walczak D, Singer J, et al. Completeness of American Cancer Registry Treatment Data: Implications for quality of care research. J Am Coll Surg. 2013:216(3):428-37.

44. Sunkara V, Hébert JR. The colorectal cancer mortality-to-incidence ratio as an indicator of global cancer screening and care. Cancer. 2015;121(10):1563-9.

45. Zauber AG. The impact of screening on colorectal cancer mortality and incidence: has it really made a difference? Digest Dis Sci. 2015;60(3):681-91.

46. Fedirko V, Tramacere I, Bagnardi V, Rota M, Scotti L, Islami F, et al. Alcoho drinking and colorectal cancer risk: an overall and dose-response metaanalysis of published studies. Ann Oncol. 2011;22(9):1958-72.

47. Moskal A, Norat T, Ferrari P, Riboli E. Alcohol intake and colorectal cancer risk: A dose-response meta-analysis of published cohort studies. Int J Cancer. 2007:120(3):664-71.

48. Bouvard V, Loomis D, Guyton KZ, Grosse Y, El Ghissassi F, Benbrahim-Tallaa $L$, et al. Carcinogenicity of consumption of red and processed meat. Lancet Oncol. 2015;16(16):1599-600.

49. Huncharek M, Muscat J, Kupelnick B. Colorectal cancer risk and dietary intake of calcium, vitamin D, and dairy products: A meta-analysis of 26,335 cases from 60 observational studies. Nutr Cancer. 2008;61(1):47-69.

50. Renehan AG, Tyson M, Egger M, Heller RF, Zwahlen M. Body-mass index and incidence of cancer: A systematic review and meta-analysis of prospective observational studies. The Lancet. 2008:371(9612):569-78.

51. Food N. Physical activity, and the Prevention of Colorectal Cancer. Continuous Update Project Report: World Cancer Research Fund. American Institute for Cancer Research. 2011.;11(2):e368

52. Harriss D, Atkinson G, Batterham A, George K, Tim Cable N, Reilly T, et al. Lifestyle factors and colorectal cancer risk (2): A systematic review and meta-analysis of associations with leisure-time physical activity. Colorect Dis. 2009:11(7):689-701.

53. Walter $\mathrm{V}$, Jansen L, Hoffmeister M, Brenner H. Smoking and survival of colorectal cancer patients: Systematic review and meta-analysis. Ann Oncol. 2014;25(8):1517-25

54. Botteri E, lodice S, Bagnardi V, Raimondi S, Lowenfels AB, Maisonneuve P. Smoking and colorectal cancer: a meta-analysis. JAMA. 2008;300(23):276578.

55. Liang PS, Chen TY, Giovannucci E. Cigarette smoking and colorectal cancer incidence and mortality: Systematic review and meta-analysis. Int J Cancer. 2009:124(10):2406-15.

56. Ang TL, Fock KM. Clinical epidemiology of gastric cancer. Singapore Med J. 2014;55(12):621.

57. Minami Y, Kawai M, Fujiya T, Suzuki M, Noguchi T, Yamanami H, et al. Family history, body mass index and survival in Japanese patients with stomach cancer: a prospective study. Int J Cancer. 2015;136(2):411-24.

58. Tsugane S, Sasazuki S. Diet and the risk of gastric cancer: review of epidemiological evidence. Gastric Cancer. 2007;10(2):75-83.

59. Park B, Shin A, Park SK, Ko K-P, Ma SH, Lee E-H, et al. Ecological study for refrigerator use, salt, vegetable, and fruit intakes, and gastric cancer. Cancer Causes Control. 2011;22(11):e1497.

60. Tsugane S. Primary prevention of gastric cancer. Nihon Rinsho Jap J Clin Med. 2012;70(10):1720-5.

This work is licensed under a Creative Commons Attribution-NonCommercial 3.0 Unported License which allows users to read, copy, distribute and make derivative works for non-commercial purposes from the material, as long as the author of the original work is cited properly. 